\title{
Dielectric Losses in Dry-Type Insulation of Medium-Voltage Power Electronic Converters
}

\section{Journal Article}

Author(s):

Guillod, Thomas (1D; Färber, Raphael (D); Rothmund, Daniel (1D; Krismer, Florian; Franck, Christian (1); Kolar, Johann W.

\section{Publication date:}

2020-09

\section{Permanent link:}

https://doi.org/10.3929/ethz-b-000377517

\section{Rights / license:}

In Copyright - Non-Commercial Use Permitted

Originally published in:

IEEE Journal of Emerging and Selected Topics in Power Electronics 8(3), https://doi.org/10.1109/jestpe.2019.2914997 


\section{ㄱㄷㄷㅗ}

Power Electronic Systems

Laboratory

(C) 2019 IEEE

IEEE Journal of Emerging and Selected Topics in Power Electronics (Early Access)

Dielectric Losses in Dry-Type Insulation of Medium-Voltage Power Electronic Converters

T. Guillod,

R. Färber,

D. Rothmund,

F. Krismer,

Ch. M. Franck,

J. W. Kolar

Personal use of this material is permitted. Permission from IEEE must be obtained for all other uses, in any current or future media, including reprinting/republishing this material for advertising or promotional purposes, creating new collective works, for resale or redistribution to servers or lists, or reuse of any copyrighted component of this work in other works.

\section{ETH}

Eidgenössische Technische Hochschule Zürich Swiss Federal Institute of Technology Zurich 


\title{
Dielectric Losses in Dry-Type Insulation of Medium-Voltage Power Electronic Converters
}

\author{
Thomas Guillod, Student Member, IEEE, Raphael Faerber, Student Member, IEEE, \\ Daniel Rothmund, Student Member, IEEE, Florian Krismer, Member, IEEE, \\ Christian M. Franck, Senior Member, IEEE, and Johann W. Kolar, Fellow, IEEE
}

\begin{abstract}
Newly available Medium-Voltage (MV) SiliconCarbide (SiC) transistors are setting new limits for the design space of MV converters. Unprecedented blocking voltages, higher switching frequencies, higher commutation speeds, lower losses, and high temperature operation can be reached, which, however, create new challenges for the electrical insulation. In particular, the dielectric losses can become significant for MV converters operated at higher switching frequencies. Moreover, the evaluation of the dielectric losses is a key element for assessing the insulation stress and for insulation diagnostics. Therefore, this paper analyzes the modeling and the computation of dielectric losses with PWM voltages. After a review of the dielectric loss mechanisms occurring in polymeric insulation materials, scalable analytical expressions are proposed for the losses produced by PWM voltages. Afterwards, the dielectric losses of a Medium-Frequency (MF) transformer, employed in a $25 \mathrm{~kW}$ MV DC-DC converter $(7 \mathrm{kV}$ to $400 \mathrm{~V})$ operated at $48 \mathrm{kHz}$, are analyzed and measured in detail. With epoxy resin, the insulation losses represent a significant share, i.e. up to $17 \%$, of the total transformer losses. As shown, the transformer performance can be significantly improved with a silicone elastomer insulation. Finally, design guidelines are provided for the selection of insulation materials.
\end{abstract}

Index Terms-Dielectrics and Electrical Insulation, Dielectric Losses, Medium-Frequency Transformers, Medium-Voltage Transformers, Silicon carbide, DC-DC power converters.

\section{INTRODUCTION}

The need to integrate renewable energy sources into the Medium-Voltage (MV) grid, to supply large DC loads from the MV grid, or to control the power flow, has led to a rapid growth of the research and industry interest for MVconnected power electronic converters [1]-[8]. The ongoing trend towards higher energy efficiencies and power densities, combined with new Silicon-Carbide (SiC) switches, promotes the use of higher switching frequencies, i.e. MediumFrequency (MF) energy conversion [2], [5], [9]. The design of such MV/MF converters is typically examined at system level [3], [4], [6], [7], [10] and component level [9], [11]-[13] while the electrical insulation is often neglected [14]-[17].

According to [18], insulation failures, in grid-connected magnetic components, are already challenging in LowFrequency (LF) systems. In [19]-[22], it is shown that the fast voltage transients generated by power converters further increase the stresses applied to a dry-type insulation system, leading to increased failure rates. In this context, the usage of new SiC switches, which show higher voltage capabilities (up tp $15 \mathrm{kV}$ ), higher switching speeds (up to $100 \mathrm{kV} / \mu \mathrm{s}$ ), and higher switching frequencies (up to $200 \mathrm{kHz}$ ), is particularly critical [1], [9], [23], [24]. Moreover, due to the high power density, elevated operating temperatures are usually occurring inside power converters. Additionally, the emergence of converter topologies featuring complex voltage waveforms, such as multi-level output voltages [15], [25] or multiple galvanically insulated ports [26]-[28], is creating new challenges for design of the electrical insulation. For these reasons, the insulation used in such power converters requires a careful examination in order to obtain a solution which accommodates the opposing requirements for compactness, long-term reliability, thermal management, and EMI performance [12], [13], [23], [29].

Literature related to the evaluation of insulation stresses and insulation diagnostics proposes the examination of partial discharges, breakdown voltages, leakage currents, field distributions, dielectric losses, etc. [15], [21], [22], [30][33]. Among these methods, the inspection of the dielectric losses is of particular interest:

- The dielectric loss densities (or changes thereof) are found to be related to the lifetime and the reliability of insulation systems, especially for MV/MF components [15], [32], [34], [35].

- The quantitative calculation of dielectric losses is possible. In contrast, the partial discharge activities or breakdown voltages are difficult to predict without extensive measurements.

- The online or offline monitoring of the insulation losses allows the detection of insulation degradation and represents a valuable diagnostic tool (e.g., quality control and preventive maintenance) [19], [34], [36], [37].

- Even if the dielectric losses are usually small compared to the total losses of a converter, this may not be the case for converters simultaneously operated at high voltages and high-frequencies [5], [10], [15], [35].

- The insulation temperature increase, due to the converter losses, can drive the dielectric itself into a highloss regime, with a subsequent thermal breakdown of the insulation [35], [38].

It should be noted that, even if the presented analysis is conducted for MV/MF applications, the same problem can appear for Low-Voltage (LV) converters operated with fastswitching Gallium-Nitride (GaN) HEMTs (above 10 MHz) [39], [40]. In such systems, due to the extreme switching frequencies, even reduced voltages (below $1 \mathrm{kV}$ ) can also produce significant insulation stresses. Accordingly, the theoretical analysis proposed in this paper is also directly applicable to LV systems operated at high-frequencies. 
To the knowledge of the authors, the exact impact of the harmonics contained in PWM voltages (compared to purely sinusoidal voltages) on the dielectric losses, up to now, has not been studied in detail. Therefore, this paper examines the computation of insulation losses in converters and is organized as follows. After recalling the definition of the dielectric losses in time and frequency domain in Section II, simple and scalable expressions are derived in Section III for their evaluation with PWM waveforms. The frequency and temperature dependences of the dielectric material properties are discussed in detail. In Section IV, the presented analysis methods are applied to a specific MV/MF transformer design. The predicted dielectric losses are successfully compared with measurements conducted on the constructed prototype. Finally, Section V presents guidelines for choosing appropriate insulation materials for MV/MF applications.

\section{Dielectric Polarization Losses}

The electrical and dielectric response of a solid insulation material depends on various microphysical processes such as polarization (e.g., electronic, atomic, and dipolar), leakage current (surface and bulk DC conductivity), charge trapping, partial discharges, etc. [32], [34], [41]. The losses occurring inside insulation materials of MV power converters are mostly linked with the dielectric polarization effects [32], [35]. The nonlinearities and additional losses which appear above the partial discharge inception voltage and at very low-frequencies (e.g., space charge migration and leakage current) are usually negligible and, therefore, not considered in this paper [20], [38], [42]. For typical materials, operating frequencies, and electric field strengths used in power converters the polarization effects are linear with respect to the electric field [38], [41], [43]. Non-linear polarization effects are mostly occurring in ferroelectric materials (e.g., ceramic capacitors), which are not used as insulation materials due to their high permittivity [38], [41]. With these assumptions, the mathematical models used for calculating the dielectric losses are presented in this Section.

\section{A. Time Domain}

As known from linear system theory, the dielectric response of a linear isotropic material is fully described with the polarization response to an electric field unit step [42], [44], [45]. This process is shown in Fig. 1 for a simple exemplary dielectric (Debye relaxation model, no leakage current) [32], [42]. The derivative of the step response (displacement field) is the impulse response (current density). At $t=0$, the impulse response goes to infinity (Dirac delta), which corresponds to a instantaneous charging of the vacuum capacitance of the considered electrode arrangement. The polarization mechanisms occurring at extreme frequencies (e.g., polarization at optical frequencies) can also be included in this quasi-instantaneous response (Dirac delta), at least for power electronic applications. It should be noted that, with the step and impulse responses, only the total losses can be determined. Without additional information on the physical nature of the polarization mech-

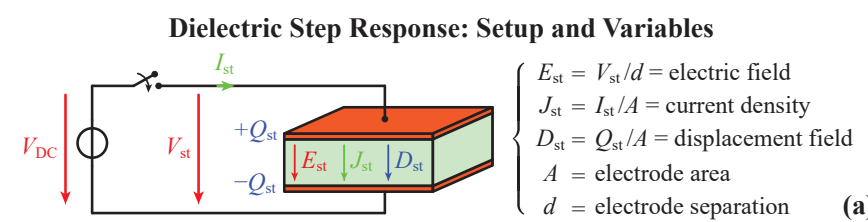

(a)
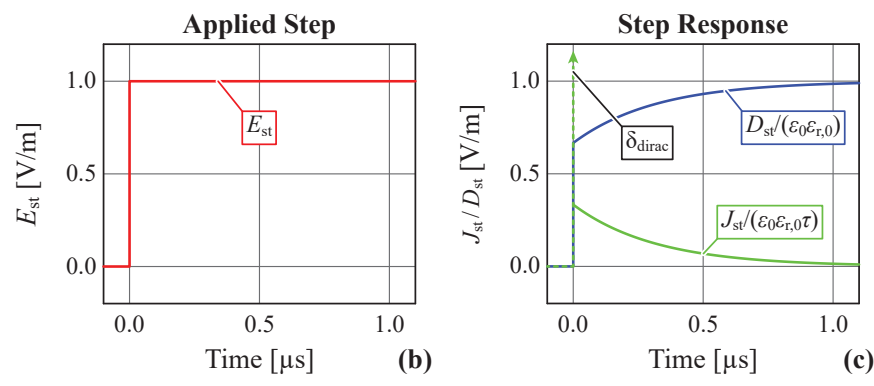

Fig. 1. (a) Variables describing the polarization response of a dielectric material to an electric field unit step. (b) Applied electric field unit step. (c) Obtained displacement field and current density responses. A Debye relaxation model (exponential charging model) is used for describing the considered exemplary material $\left(\varepsilon_{r, 0}=3.0, \varepsilon_{r, \infty}=2.0, \tau=320 \mathrm{~ns}\right)$ [32], [42].

anisms (e.g., transfer dynamics between the dipoles and their molecular environment), an instantaneous dielectric loss rate cannot be specified.

The polarization response to an arbitrary voltage excitation can be obtained by convoluting the applied electric field with the impulse response [32], [45]:

$$
D(t)=\int_{0}^{\infty} J_{\text {st }}\left(t^{\prime}\right) E\left(t-t^{\prime}\right) \mathrm{d} t^{\prime}, \quad J(t)=\frac{\partial D(t)}{\partial t},
$$

where $E$ is the applied electric field, $J_{\text {st }}$ the impulse response, $D$ the obtained displacement field, and $J$ the obtained current density. Then, the input power per unit volume $(p)$ and the supplied energy density $(w)$ can be written as

$$
p(t)=E(t) J(t), \quad w(t)=\int_{0}^{t} p\left(t^{\prime}\right) \mathrm{d} t^{\prime} .
$$

The time domain convolution is illustrated in Fig. 2 for trapezoidal electric field pulses, as resulting from PWM voltages. The dielectric response $(D, J, p$, and $w)$ contains contributions from the vacuum permittivity (instantaneous response and lossless) and from the material polarization (linked to the dielectric susceptibility, delayed response, and lossy). The dielectric losses can be obtained by making the energy balance between the supplied energy and the energy stored in the field, cf. Fig. 2(e). In analogy to magnetic losses, the dielectric losses can also be extracted from the area between the electric field and the displacement field i.e. the hysteresis curve, cf. Fig. 2(f).

Despite its conceptual clarity, the computation of dielectric losses in time domain is often problematic since it involves a convolution integral. Also, the step and impulse responses of the insulation materials are often not directly available. Moreover, the numerical simulation of the electric field in time domain is computationally intensive (e.g., FEM). For these reasons, it is preferable, especially for periodic excitations, to compute the dielectric losses in frequency domain. 



Fig. 2. Material response to (a) electric field pulses: (b) displacemen field, (c) current density, (d) input power, (e) supplied energy, and (f) hysteresis cycle. The different variables are, whenever possible, split into two parts: vacuum permittivity and material polarization. A Debye relaxation model (exponential charging model) is used for describing the considered exemplary material $\left(\varepsilon_{r, 0}=3.0, \varepsilon_{r, \infty}=2.0, \tau=320 \mathrm{~ns}\right)$ [32], [42].

\section{B. Frequency Domain}

The response of a linear isotropic dielectric can be described with its complex permittivity and conductivity. The following relationships can be defined [32]:

$$
\begin{aligned}
& J(f)=J_{\mathrm{c}}(f)+J_{\mathrm{d}}(f)=\sigma E(f)+\mathrm{j} 2 \pi f \varepsilon(f) E(f), \\
& \varepsilon(f)=\varepsilon_{0} \varepsilon_{\mathrm{r}}(f)=\varepsilon_{0}\left(\varepsilon_{\mathrm{r}}^{\prime}(f)-\mathrm{j} \varepsilon_{\mathrm{r}}^{\prime \prime}(f)\right),
\end{aligned}
$$

where $f$ is the frequency, $J$ the current density (due to both free and bound charges), $E$ the electric field, $\sigma$ the conductivity, and $\varepsilon(f)$ the permittivity. The permittivity is obtained by the following Fourier transform [32], [45]:

$$
\varepsilon_{\mathrm{r}}(f)=\varepsilon_{\mathrm{r}}(\infty)+\frac{1}{\varepsilon_{0} E_{0}} \int_{0}^{\infty} J_{\mathrm{st}}(t) \mathrm{e}^{-\mathrm{j} 2 \pi f t} \mathrm{~d} t
$$

where $J_{\text {st }}$ is the displacement current density response to an electric field unit step (cf. Fig. 1(a)). The fact that the frequency domain permittivity is the Fourier transform of a real function imposes some restrictions on $\varepsilon_{\mathrm{r}}(f)$. Moreover, the polarization step response respects causality (equal to zero for $t<0$ ). These conditions are expressed by the
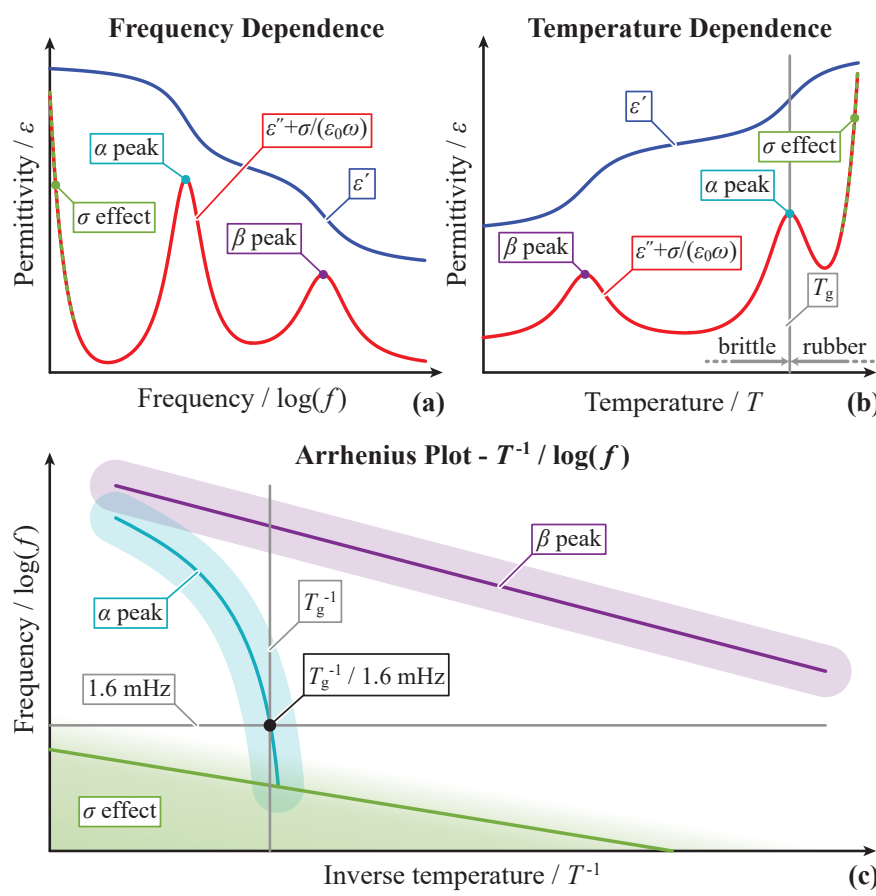

Fig. 3. (a) Frequency and (b) temperature dependences of the complex permittivity for an exemplary polymeric insulation material. (c) Arrhenius plot showing the frequency and temperature dependences of the loss peaks. The effects of the $\alpha$ relaxation, $\beta$ relaxation, and conductivity $(\sigma)$ are shown, as well as the definition of the glass transition temperature, $T_{\mathrm{g}}$.

Kramers-Kronig relations which link the real and imaginary parts of the permittivity [32], [42]:

$$
\begin{aligned}
& \varepsilon_{\mathrm{r}}^{\prime}(f)=\varepsilon_{\mathrm{r}}^{\prime}(\infty)+\frac{2}{\pi} \mathscr{P} \int_{0}^{\infty} \frac{f^{\prime} \varepsilon_{\mathrm{r}}^{\prime \prime}\left(f^{\prime}\right)}{f^{\prime 2}-f^{2}} \mathrm{~d} f^{\prime}, \\
& \varepsilon_{\mathrm{r}}^{\prime \prime}(f)=-\frac{2}{\pi} \mathscr{P} \int_{0}^{\infty} \frac{f\left(\varepsilon_{\mathrm{r}}^{\prime}\left(f^{\prime}\right)-\varepsilon_{\mathrm{r}}^{\prime}(\infty)\right)}{f^{\prime 2}-f^{2}} \mathrm{~d} f^{\prime},
\end{aligned}
$$

where $\mathscr{P}$ denotes the Cauchy principal value of an integral (handling of the singularity). If the variation of $\varepsilon_{\mathrm{r}}(f)$ is moderate (in a given frequency range), the following approximation of (7) can be derived [42], [45]:

$$
\varepsilon_{\mathrm{r}}^{\prime \prime}(f) \approx-\frac{\pi}{2} \frac{\partial \varepsilon_{\mathrm{r}}^{\prime}(f)}{\partial \ln (f)} .
$$

This equation states that a decrease of the real part of the permittivity in a given frequency range will produce a loss peak (imaginary part) in the same frequency range. Compared to (7), this approximation is much simpler: no integral over the complete spectrum, no singularity, and applicable locally.

Fig. 3(a) depicts the frequency dependence (at a fixed temperature) of the complex permittivity for an exemplary polymeric insulation material. With increasing frequency, the dipoles successively lose their ability to follow the external electric field and the real part (and magnitude) of the permittivity drops [32], [41], [46]. It can be seen that the derivative of the real part (in a logarithmic frequency scale) is approximately proportional to the imaginary part (cf. (8)). Two typical loss peaks, corresponding to different types of 
relaxing dipoles, are shown: segmental motion of polymer chains ( $\alpha$ relaxation) and orientation of local dipoles ( $\beta$ relaxation). Other loss peaks may occur (e.g., $\gamma$ relaxation and MWS mode) but are less relevant for the frequency range used by power electronic converters [41], [46]. Towards the lower end of the frequency spectrum (typically well below $50 \mathrm{~Hz}$ ), the loss due to the DC conductivity (leakage current) begins to dominate the imaginary part of the (effective) permittivity (cf. (3)).

Fig. 3(b) shows a typical temperature dependence (at a fixed frequency) of the complex permittivity. The real part of the permittivity increases with temperature due to the higher dipole mobility [41], [46]. Again, contributions from discrete relaxation modes ( $\alpha$ and $\beta$ relaxations) and DC conductivity are present (in reverse order compared to the frequency dependence). The $\alpha$ relaxation peak defines the glass transition temperature of the material, $T_{\mathrm{g}}$, where the material is going from a brittle glassy state to a viscoelastic rubbery state, with a characteristic drop in the polymer's elastic modulus [46]. The glass transition temperature is typically measured at very low frequencies, where $f=1.6 \mathrm{mHz}$ represents the prevalent choice [46].

Fig. 3(c) shows the corresponding Arrhenius plot where the frequency and temperature dependences of the loss peaks are simultaneously illustrated. Usually, the $\alpha$ and $\beta$ peaks merge into a single peak at high-frequency [46]. At low-frequencies or high temperatures, the leakage current dominates over the polarization current.

It should be noted that the AC losses can be arbitrarily split between $\sigma$ and $\varepsilon_{\mathrm{r}}^{\prime \prime}$ while the DC losses can only be represented with $\sigma$ (cf. (3)). Moreover, with typical AC dielectric spectroscopy measurements, only the total losses are measured and the conduction and polarization losses cannot be split [34], [37]. Usually, $\sigma$ is set with the DC conductivity and is accepted to be frequencyindependent. Then, $\varepsilon_{\mathrm{r}}^{\prime \prime}$ is chosen such that $\sigma+2 \pi f \varepsilon_{\mathrm{r}}^{\prime \prime}$ matches the measured AC losses. However, the conduction losses in the insulation of power converters are usually negligible (compared to the polarization losses). Therefore, only the polarization losses are considered in this paper.

\section{Loss Computation}

With the help of the complex permittivity, it is straightforward to express the time-averaged losses of an insulation system excited with a periodic electric field as

$$
P=\iiint_{\mathrm{V}}\left(\sum_{n=1}^{\infty} \varepsilon_{0} \varepsilon_{\mathrm{r}}^{\prime \prime}\left(n f_{\mathrm{s}}\right)\left(2 \pi n f_{\mathrm{s}}\right) E_{n, \mathrm{RMS}}^{2}\right) \mathrm{dV},
$$

where $\mathrm{V}$ is the insulation volume, $f_{\mathrm{s}}$ the fundamental frequency, and $E_{n, \mathrm{RMS}}$ the RMS Fourier series coefficients of the electric field norm. In case of a homogeneous and isothermal dielectric placed between an arbitrary number of electrodes, the expression for the losses can be simplified to

$$
P=\sum_{n=1}^{\infty} \varepsilon_{\mathrm{r}}^{\prime \prime}\left(n f_{\mathrm{s}}\right)\left(2 \pi n f_{\mathrm{s}}\right) \varphi_{n, \mathrm{RMS}}^{\top} C_{0} \varphi_{n, \mathrm{RMS}},
$$

where $C_{0}$ is the vacuum capacitance matrix (describing the geometry) and $\varphi_{n \text {, RMS }}$ is a vector containing the RMS
Fourier series coefficients of the potentials applied to the different electrodes. The matrix is computed with $\varepsilon_{0}$ such that $Q=C_{0} \varphi$, where $Q$ and $\varphi$ are vectors containing the charges and the potentials (with respect to the reference potential $0 \mathrm{~V}$ ) of the different electrodes.

The aforementioned formulas make use of the complex permittivity. However, for a given material, the absolute value of the permittivity $\left(\varepsilon_{\mathrm{r}}\right)$ is often given (e.g., norms and datasheets) together with the loss tangent or dissipation factor $(\tan \delta)$. Equivalent circuits consisting of the series ( $C_{\mathrm{ESR}}$ and $R_{\mathrm{ESR}}$ ) or parallel ( $C_{\mathrm{EPR}}$ and $R_{\mathrm{EPR}}$ ) connection of a capacitor and a resistor are also common. These quantities are related to the real and imaginary part of the complex permittivity as follows:

$$
\begin{aligned}
& \tan \delta=\frac{\varepsilon_{\mathrm{r}}^{\prime \prime}}{\varepsilon_{\mathrm{r}}^{\prime}}, \\
& C_{\mathrm{ESR}}=\frac{\varepsilon_{\mathrm{r}}^{\prime \prime 2}+\varepsilon_{\mathrm{r}}^{\prime 2}}{\varepsilon_{\mathrm{r}}^{\prime}} C_{0} \stackrel{\varepsilon_{\mathrm{r}}^{\prime} \gg \varepsilon_{\mathrm{r}}^{\prime \prime}}{\approx} \varepsilon_{\mathrm{r}}^{\prime} C_{0}, \\
& R_{\mathrm{ESR}}=\frac{\varepsilon_{\mathrm{r}}^{\prime \prime}}{\varepsilon_{\mathrm{r}}^{\prime \prime 2}+\varepsilon_{\mathrm{r}}^{\prime 2}} \frac{1}{2 \pi f C_{0}} \stackrel{\varepsilon_{\mathrm{r}}^{\prime} \gg \varepsilon_{\mathrm{r}}^{\prime \prime}}{\approx} \frac{\varepsilon_{\mathrm{r}}^{\prime \prime}}{\varepsilon_{\mathrm{r}}^{\prime}} \frac{1}{2 \pi f C_{\mathrm{ESR}}}, \\
& C_{\mathrm{EPR}}=\varepsilon_{\mathrm{r}}^{\prime} C_{0}, \\
& R_{\mathrm{EPR}}=\frac{1}{\varepsilon_{\mathrm{r}}^{\prime}} \frac{1}{2 \pi f C_{0}}=\frac{\varepsilon_{\mathrm{r}}^{\prime}}{\varepsilon_{\mathrm{r}}^{\prime \prime}} \frac{1}{2 \pi f C_{\mathrm{EPR}}} .
\end{aligned}
$$

The loss tangent is a figure of merit for capacitors (losses compared to the stored energy). Since energy storage is not a desirable property of electrical insulation, the loss tangent is not a figure of merit for insulation materials. Models based on the series or parallel equivalent circuits are massively frequency-dependent even for a constant complex permittivity. The approximation of a frequency-independent equivalent circuit, which is common for the computation of capacitor losses in case of constant frequency sinusoidal operation, is invalid for the dielectric losses of insulation materials with PWM voltages (due to the broad voltage spectrum). This would lead to a massive overestimation ( $C_{\mathrm{ESR}}$ and $R_{\mathrm{ESR}}$ ) or underestimation $\left(C_{\mathrm{EPR}}\right.$ and $\left.R_{\mathrm{EPR}}\right)$ of the dielectric losses. It should also be noted that the instantaneous losses obtained with the series or parallel equivalent circuits do not feature any physical meaning (only the average losses should be considered). For these reasons, the complex permittivity representation in frequency domain is used in this paper.

\section{Dielectric Losses With PWM Voltages}

In steady state operation, typical power converters generate PWM voltages which are also applied to the insulation. Expression (10) allows the calculation of the dielectric losses by means of numerical computations. It is, however, very difficult to separate the influence of the different parameters since the frequency-dependent material parameter $\varepsilon_{\mathrm{r}}^{\prime \prime}(f)$ and the amplitude of the voltage harmonic components appear in the product term of the infinite sum.

In order to find a closed-form expression for the losses produced by PWM voltages, a constant value of $\varepsilon_{\mathrm{r}}^{\prime \prime}(f)$ is first considered in Subsection III-A, which, according to [43] is an admissible assumption for certain insulation materials. This holds true for low-loss polymeric materials (e.g., PTFE and PP) for which no significant relaxation peaks occur within the 

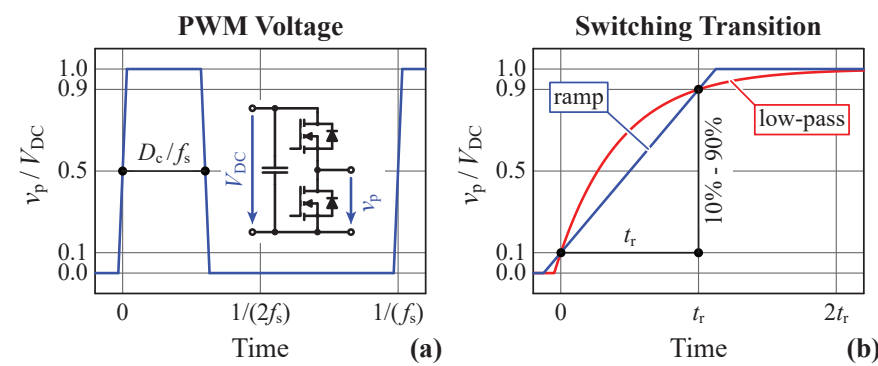

Fig. 4. (a) PWM signal with a constant duty cycle. (b) Two different approximations of the switching transition (rise time $t_{\mathrm{r}}$ ): the ramp function and the step response of a first-order low-pass filter.

frequency band excited by typical PWM voltages. With this hypothesis, $\varepsilon_{\mathrm{r}}^{\prime \prime}(f)$ can be taken out of the sum (10), allowing the decoupling of the impact of the material properties from the applied excitation. Afterwards, in Subsection III-B, the frequency dependence of the permittivity is analyzed. Finally, Subsection III-C and Subsection III-D explain the implications of such frequency dependences on the dielectric losses.

\section{A. PWM with Frequency-Independent Materials}

An insulation system with two electrodes is considered and a PWM voltage is applied, as illustrated in Fig. 4(a). The switching transitions are shown in Fig. 4(b). The commonly used ramp function generates a spectrum with side lobes $(\sin (x) / x$ shaped harmonics) and, therefore, is complex to analyze in frequency domain. However, the step response of a first-order low-pass filter features a similar spectrum which can be described with a simpler formula. For this reason, the step response of the following low-pass filter is used:

$$
G_{\mathrm{lp}}(f)=\frac{1}{1+\mathrm{j} \frac{f}{f_{\mathrm{c}}}}, \quad f_{\mathrm{c}}=\frac{\ln \left(\frac{0.9}{0.1}\right)}{2 \pi t_{\mathrm{r}}},
$$

where $t_{\mathrm{r}}$ is the $10 \%-90 \%$ rise time, cf. Fig. $4(\mathrm{~b})$. The associated harmonic components (Fourier series, without DC component) of the PWM voltage can be computed as

$$
V_{n, \mathrm{RMS}}=\left(\frac{\sqrt{2}}{\pi} \frac{\left|\sin \left(\pi n D_{\mathrm{c}}\right)\right|}{n} V_{\mathrm{DC}}\right)\left|G_{\mathrm{lp}}\left(n f_{\mathrm{s}}\right)\right|,
$$

where $D_{\mathrm{c}}$ is the duty cycle and $V_{\mathrm{DC}}$ the amplitude. With the voltage harmonics, the spectral components of the losses (cf. (10)) and the total (time averaged) losses can be computed:

$$
\begin{aligned}
P_{n} & =\left(\varepsilon_{\mathrm{r}}^{\prime \prime} C_{0}\right)\left(2 \pi n f_{\mathrm{s}}\right) V_{n, \mathrm{RMS}}^{2} . \\
P_{n, \mathrm{c}} & =\sum_{n^{\prime}=1}^{n} P_{n^{\prime}}, \quad P=\lim _{n \rightarrow \infty} P_{n, \mathrm{c}}=\sum_{n^{\prime}=1}^{\infty} P_{n^{\prime}},
\end{aligned}
$$

where $f_{\mathrm{s}}$ is the switching frequency, $P_{n}$ the spectral losses, $P_{n, \mathrm{c}}$ the partial losses of the first $n$ harmonics, and $P$ the total losses. The assumption $\varepsilon_{\mathrm{r}}^{\prime \prime}(f)=$ const. is made.

Fig. 5 depicts the voltage harmonics (cf. (17)), the loss spectral components (cf. (18)), and the cumulative sum of the losses (cf. (19)). The voltage and power harmonics are proportional to $\sim 1 / n$ for frequencies below the corner
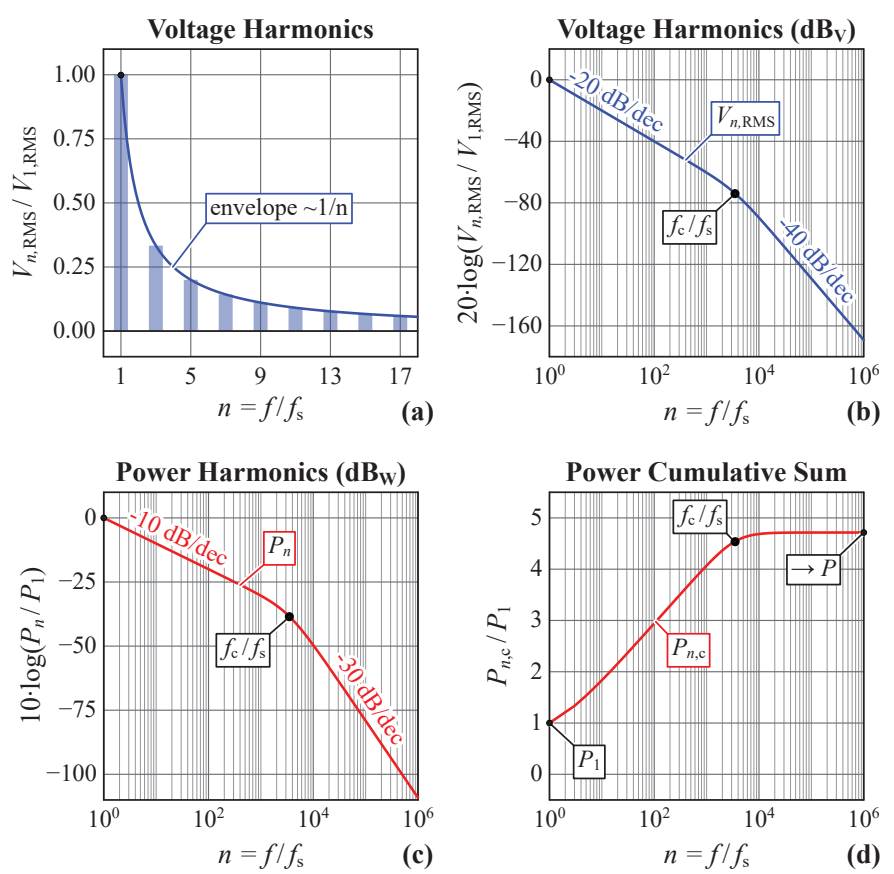

Fig. 5. (a) Voltage harmonics for a PWM signal with a constant duty cycle (representation limited to the first harmonics), (b) envelope of the voltage harmonics (in $\mathrm{dB}_{\mathrm{V}}$ ), (c) envelope of the power loss harmonics (in $\mathrm{dB}_{\mathrm{W}}$ ), and (d) cumulative sum of the power harmonics. The following parameters are used: $f_{\mathrm{s}}=1 \mathrm{kHz}, t_{\mathrm{r}}=100 \mathrm{~ns}$, and $D_{\mathrm{c}}=50 \%$. With the chosen duty cycle, no even harmonics are present.

frequency of the low-pass filter. Above the corner frequency, the voltage and power harmonics are proportional to $1 / n^{2}$ and $\sim 1 / n^{3}$, respectively. Therefore, the sum (19) only converges above the corner frequency [47].

This implies that an infinitesimally short switching transition would lead to infinite losses. However, this is only true with the assumption of a frequency-independent complex permittivity, which is nonphysical for very large frequency ranges (cf. (8)). Nevertheless, the aforementioned results indicate that a model of the switching transition is required for evaluating the dielectric losses with PWM voltages (cf. (16)). Moreover, the sum $P$ is much greater than the fundamental harmonic $P_{1}$, showing that a fundamental frequency analysis is invalid.

The calculation of (19) is computationally intensive since it requires the summation of many harmonics (cf. Fig. 5(d)). This holds especially true for the cases where the electric field patterns are obtained from numerical simulations and the summation is performed on a meshed geometry. Therefore, a closed-form approximation is proposed (with the hypothesis $\varepsilon_{\mathrm{r}}^{\prime \prime}(f)=$ const.):

$$
\begin{aligned}
P & \approx \lambda P_{1}, \\
P_{1} & =\left(\varepsilon_{\mathrm{r}}^{\prime \prime} C_{0}\right)\left(2 \pi f_{\mathrm{s}}\right)\left(\frac{\sqrt{2}}{\pi} V_{\mathrm{DC}}\right)^{2}, \\
\lambda & =\frac{1}{2} \ln \left(2 \mathrm{e}^{\gamma} \frac{f_{\mathrm{c}}}{f_{\mathrm{s}}} \sin \left(\pi D_{\mathrm{c}}\right)\right),
\end{aligned}
$$

where $P_{1}$ expresses the losses at the fundamental frequency (with $D_{\mathrm{c}}=50 \%$ ). The correction factor $\lambda$ takes into account the duty cycle and the harmonics. The constant 



Fig. 6. (a) Impact of the switching frequency, the rise time, and (b) the duty cycle on the dielectric losses. The excitation is a PWM signal with a constant duty cycle. All the power values are normalized with respect to $\varepsilon_{\mathrm{r}}^{\prime \prime} C_{0} V_{\mathrm{DC}}^{2}$ while the used parameters are given in the figures.

$\gamma \approx 0.58$ is the Euler-Mascheroni constant. The derivation of (20) requires elaborate calculations, which are given in Appendix A. The accuracy of the approximation is evaluated in Appendix E for different frequencies, duty cycles, and switching speeds. It is found that the approximation is highly accurate ( $1 \%$ error). The error is also small ( $4 \%$ error) if a ramp function is used instead of the low-pass filter step response for modeling the switching transition (cf. Fig. 4(b)). This indicates that an exact model of the switching transition is not necessary and that the model used in (16) is sufficient. Moreover, this approximation can be applied to PWM signals with variable duty cycle (e.g., sinusoidal modulation and multi-level inverters) by means of local duty cycle averaging, as shown in [14].

Fig. 6 depicts the results obtained with (20) for different rise times, switching frequencies, and duty cycles for typical values encountered in MV converters. It can be seen that the impact of the voltage $\left(P \sim V_{\mathrm{DC}}^{2}\right)$ and frequency $(P \sim$ $f_{\mathrm{s}} \ln \left(\right.$ const. $\left.\left./ f_{\mathrm{s}}\right)\right)$ are very strong while the losses increase only logarithmically with respect to the reciprocal of the rise time $\left(P \sim \ln\right.$ (const./ $\left.\left.t_{\mathrm{r}}\right)\right)$. The influence of the duty cycle on the losses is almost negligible in the practically relevant range $\left(D_{\mathrm{c}} \in[10 \%, 90 \%]\right)$.

\section{B. Frequency-Dependent Materials}

In the previous Subsection, a constant $\varepsilon_{\mathrm{r}}^{\prime \prime}(f)$ has been assumed. However, due to the coupling between the real and imaginary part of the permittivity (Kramers-Kronig relations, cf. (8)), this approximation cannot be accurate in a wide frequency range. The fact that the insulation of power converters is subject to a wide range of operating temperatures and frequencies increases the probability of encountering loss peaks (cf. Fig. 3).

In this paper, at first, an epoxy resin is considered since this a common insulation material for potting MV components. This resin is an unfilled epoxy ("vonRoll Damisol 3418") with a high glass transition temperature $\left(T_{\mathrm{g}}=136^{\circ} \mathrm{C}\right)$ [36], [49]. Due to the high glass transition temperature, this epoxy features low losses in a wide temperature range (cf. Fig. 3).

The complex permittivity is typically measured with a dielectric spectroscopy setup. A voltage is applied to the dielectric and the current is measured. Then, the complex
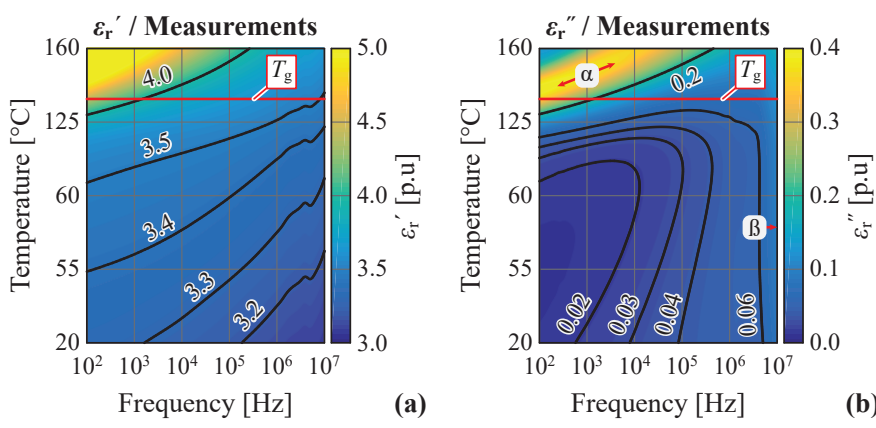

Fig. 7. (a) Measured $\varepsilon_{\mathrm{r}}^{\prime}(f, T)$ and (b) $\varepsilon_{\mathrm{r}}^{\prime \prime}(f, T)$ for the epoxy resin "vonRoll Damisol 3418" [48]. The glass transition temperature $\left(T_{\mathrm{g}}=136^{\circ} \mathrm{C}\right)$ is indicated. The $\alpha$ relaxation peak and the beginning of the $\beta$ relaxation are shown.
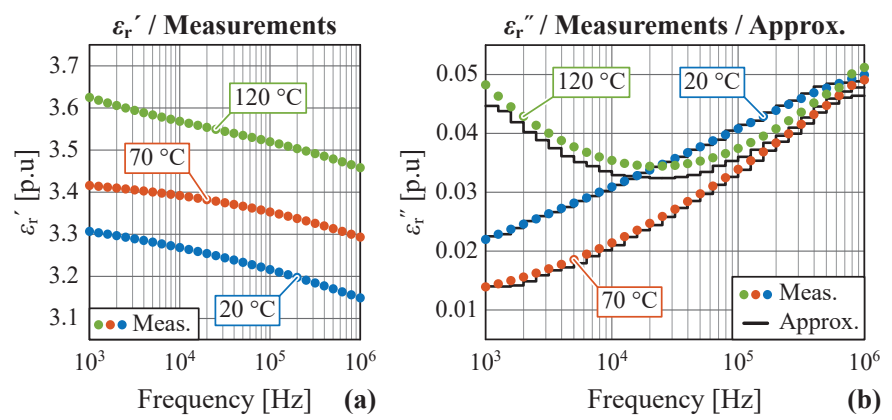

Fig. 8. (a) Measured $\varepsilon_{\mathrm{r}}^{\prime}(f)$ and (b) $\varepsilon_{\mathrm{r}}^{\prime \prime}(f)$ at different temperatures for the epoxy resin "vonRoll Damisol 3418" [48]. An approximation of $\varepsilon_{\mathrm{r}}^{\prime \prime}(f)$, computed with the Kramers-Kronig approximation (8), is also shown. The selected frequencies and temperatures are located below the $\alpha$ and $\beta$ peaks (cf. Fig. 3).

impedance is computed and the complex permittivity can be extracted. Such measurements are intrinsically illconditioned due to the low dissipation factor, i.e. the phase shift between the voltage and the current is almost $90^{\circ}$. Therefore highly accurate impedance measurement setups, which are optimized and/or calibrated for capacitive loads, are required [36], [37]. Fig. 7 shows the measured complex permittivity for the selected resin. The measurements are done with a disc-shaped specimen using a "Novocontrol Alpha-A" high precision dielectric spectroscopy system [48]

The $\alpha$ relaxation peak (at the glass transition temperature) and the beginning of the $\beta$ relaxation peak (peak above $10 \mathrm{MHz}$ ) can be identified (cf. Fig. 3). The $\alpha$ relaxation peak is very large and corresponding large losses result if the epoxy resin is operated near the glass transition temperature. It is therefore obvious that the approximation of a constant complex permittivity can only be valid in a limited frequency and temperature range.

The Kramers-Kronig relations can be applied to the measured data. Between the relaxation peaks, where the permittivity curve is flat, the approximation (8) of the Kramers-Kronig integral (7) can be used. Fig. 8 shows the correlation between the real and imaginary part of the permittivity and the curves reconstructed with (8). A detailed analysis of the accuracy of the approximation of the KramersKronig relations is presented in Appendix D and it is found that the approximation is valid between the loss peaks $(9 \%$ error). 


\section{Upper Bound on the Dielectric Losses}

In Subsection III-A, it has been shown that the model of a frequency-independent permittivity diverges for infinite switching speeds. With the results presented in Subsection III-B, this problem can be eliminated and an upper bound for the dielectric losses can be derived.

A single (non-periodic) voltage transition (step response of a first-order low-pass filter, cf. Fig. 4(b)) is considered. With the Fourier transformation, the dissipated energy can be calculated in frequency domain:

$$
W=C_{0} V_{\mathrm{DC}}^{2}\left(\frac{1}{\pi} \int_{0}^{\infty} \varepsilon_{\mathrm{r}}^{\prime \prime}(f) \frac{1}{f} \frac{f_{\mathrm{c}}^{2}}{f^{2}+f_{\mathrm{c}}^{2}} \mathrm{~d} f\right) .
$$

The derivation of (23) is given in Appendix B. For slow switching transition (cf. (16)), the integral converges to zero:

$$
\lim _{t_{\mathrm{r}} \rightarrow \infty} W=0 \text {. }
$$

With the Kramers-Kronig relations (cf. (6)), the expression (23) can be rewritten as

$$
W=C_{0} V_{\mathrm{DC}}^{2}\left(\frac{\varepsilon_{\mathrm{r}}^{\prime}(0)-\varepsilon_{\mathrm{r}}^{\prime}(\infty)}{2}-\frac{1}{\pi} \int_{0}^{\infty} \varepsilon_{\mathrm{r}}^{\prime \prime}(f) \frac{f}{f^{2}+f_{\mathrm{c}}^{2}} \mathrm{~d} f\right) .
$$

The derivation of (25) is also given in Appendix B. For a switching transition with an infinite switching speed (cf. (16)), the integral converges to zero. This implies that the first term of the equation represents an upper bound for the dielectric losses [45]:

$$
\lim _{t_{\mathrm{r}} \rightarrow 0} W=C_{0} V_{\mathrm{DC}}^{2} \frac{\varepsilon_{\mathrm{r}}^{\prime}(0)-\varepsilon_{\mathrm{r}}^{\prime}(\infty)}{2} .
$$

However, with periodic PWM voltages, this upper bound is usually not reached due to the limited switching speed of the semiconductors. Additionally, a periodic PWM signal does not excite frequencies below the switching frequency, which is not the case for a single (non-periodic) switching transition. Therefore, this upper bound is only of theoretical interest and cannot be used for computing the dielectric losses of insulation systems. An approximation taking into account the finite switching speed and the presence of a finite fundamental period is presented in the next Subsection.

\section{PWM with Frequency-Dependent Materials}

The approaches considered in Subsection III-A (cf. (20)) and Subsection III-B can be combined for extracting a closedform approximation of the dielectric losses produced by frequency-dependent materials under PWM voltages:

$$
\begin{aligned}
& P=\left(\lambda_{1}+\lambda_{2}\right) P_{1}, \\
& P_{1}=\left(\varepsilon_{\mathrm{r}}^{\prime \prime}\left(f_{\mathrm{s}}\right) C_{0}\right)\left(2 \pi f_{\mathrm{s}}\right)\left(\frac{\sqrt{2}}{\pi} V_{\mathrm{DC}}\right)^{2}, \\
& \lambda_{1}=\frac{1}{2} \ln \left(2 \mathrm{e}^{\gamma} \sin \left(\pi D_{\mathrm{c}}\right)\right) . \\
& \lambda_{2}=\frac{1}{2 \varepsilon_{\mathrm{r}}^{\prime \prime}\left(f_{\mathrm{s}}\right)} \underbrace{\int_{\ln \left(f_{\mathrm{s}}\right)}^{\ln \left(f_{\mathrm{c}}\right)} \varepsilon_{\mathrm{r}}^{\prime \prime}(f) \mathrm{d}(\ln (f)),}_{\varepsilon_{\mathrm{r}, \text { int }}^{\prime \prime}}
\end{aligned}
$$
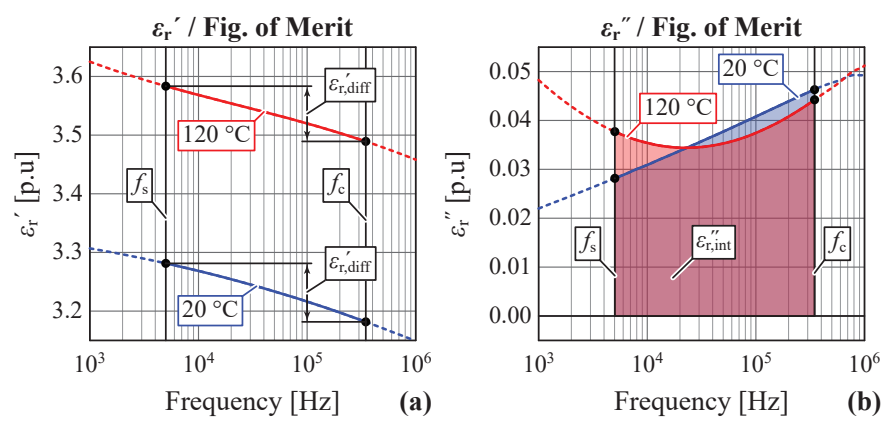

Fig. 9. (a) Measured $\varepsilon_{\mathrm{r}}^{\prime}(f)$ and (b) $\varepsilon_{\mathrm{r}}^{\prime \prime}(f)$ for the epoxy resin "vonRoll Damisol 3418". The difference $\varepsilon_{\text {r,diff }}^{\prime}$ (cf. (34)) and the area $\varepsilon_{\mathrm{r} \text {,int }}^{\prime \prime}$ (cf. (30)), which are figures of merit for the losses, are shown.

where $P_{1}$ represents the losses at the fundamental frequency. The correction factors $\lambda_{1}$ and $\lambda_{2}$ describe the impact of the duty cycle, the harmonics, and the frequency dependence of the material parameters. The derivation of (27) is given in Appendix C. The proposed formula requires the measurement of $\varepsilon_{\mathrm{r}}^{\prime \prime}(f)$ for $f \in\left[f_{\mathrm{s}}, f_{\mathrm{c}}\right]$ (cf. (16) and (17)), which is not always available and difficult to measure (due to $\varepsilon_{\mathrm{r}}^{\prime} \gg \varepsilon_{\mathrm{r}}^{\prime \prime}$ ) [34], [37], [50]. However, with the Kramers-Kronig relations (cf. (8)), the approximation (30) can be rewritten as

$$
\begin{aligned}
P & =\left(\lambda_{1}+\lambda_{2}\right) P_{1}, \\
P_{1} & =\left(\left(-\left.\frac{\pi}{2} \frac{\partial \varepsilon_{\mathrm{r}}^{\prime}(f)}{\partial \ln (f)}\right|_{f_{\mathrm{s}}}\right) C_{0}\right)\left(2 \pi f_{\mathrm{s}}\right)\left(\frac{\sqrt{2}}{\pi} V_{\mathrm{DC}}\right)^{2}, \\
\lambda_{1} & =\frac{1}{2} \ln \left(2 \mathrm{e}^{\gamma} \sin \left(\pi D_{\mathrm{c}}\right)\right) . \\
\lambda_{2} & =\frac{\pi}{4 \varepsilon_{\mathrm{r}}^{\prime \prime}\left(f_{\mathrm{s}}\right)} \underbrace{\left(\varepsilon_{\mathrm{r}}^{\prime}\left(f_{\mathrm{s}}\right)-\varepsilon_{\mathrm{r}}^{\prime}\left(f_{\mathrm{c}}\right)\right)}_{\varepsilon_{\mathrm{r}, \text { diff }}^{\prime}} .
\end{aligned}
$$

The area $\varepsilon_{\text {rint }}^{\prime \prime}$ (cf. (30)) approximates the summation over frequencies of the dielectric losses (cf. (19)) and therefore, is a figures of merit for the losses generated by a dielectric material with PWM voltages. The integral can be combined with the derivative present of the Kramers-Kronig approximation (cf. (8)) leading to an even simpler figure of merit: the difference $\varepsilon_{\text {rdiff }}^{\prime}$ (cf. (34)). Fig. 9 illustrates how these figures of merit can be used for comparing the losses at different temperatures. In addition, they are useful for comparing the losses between different materials.

The accuracy of the approximations is evaluated in Appendix E for different frequencies, duty cycles, switching speeds, and temperatures. The approximations are found to be valid for the selected resin: $8 \%$ error with (27) and $16 \%$ error with (31). If the frequency dependence of the material parameters is neglected (cf. (20)), the error is greater than $100 \%$, indicating that the frequency dependence of the permittivity must be considered for accurate computations.

\section{CASE Study: MV/MF Transformer}

With the help of the proposed approximations, the dielectric losses of different components can be examined. Since it has been identified that the stresses are particularly 


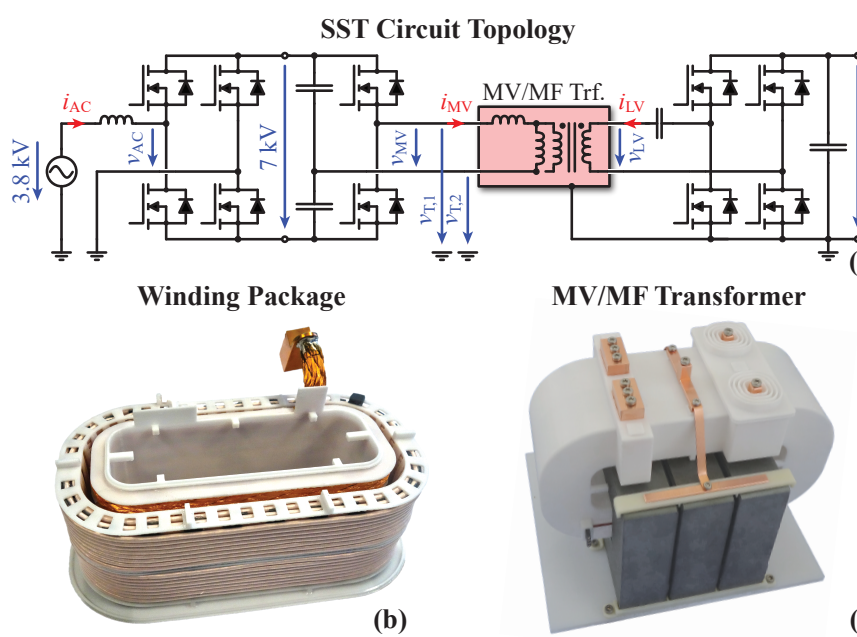

(c)

Fig. 10. (a) Considered SST (AC-DC and isolated DC-DC converters) where the MV/MF transformer is highlighted. (b) MV/MF transformer windings (before vacuum potting). (c) Constructed MV/MF transformer prototype.
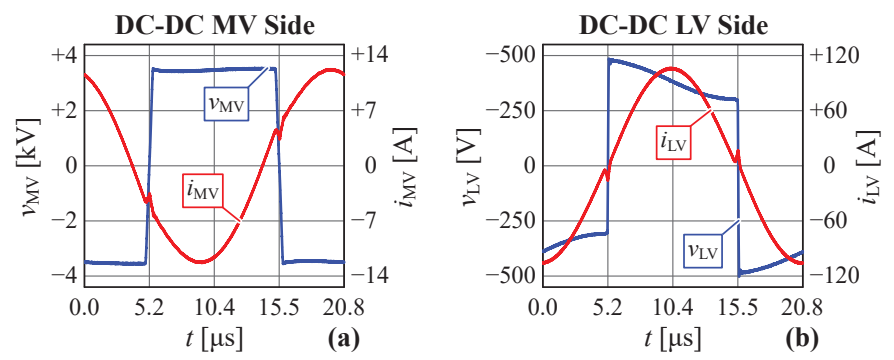

Fig. 11. Measured voltages and currents for the (a) MV side and (b) LV side of the MV/MF transformer. The series-resonant DC-DC converter is operated at the switching frequency.

high for MV/MF transformers operated inside MV DC-DC converters, such a transformer has been selected for this case study [13], [14], [17], [23], [24].

\section{A. Transformer Design}

A $25 \mathrm{~kW}$ single-phase MV SiC Solid-State Transformer (SST) interfacing a $3.8 \mathrm{kV}$ (RMS, phase-to-ground) AC grid to a $400 \mathrm{~V}$ DC bus is considered [1], [4], [5], [14]. Fig. 10(a) shows the circuit topology, which consists of a full-bridge AC-DC rectifier coupled with an insulated series-resonant DC-DC converter. The AC-DC and the DC-DC converters share a common $7 \mathrm{kV}$ DC bus and the usage of $10 \mathrm{kV}$ SiC MOSFETs allows the realization of both converters with a single-stage structure. Zero Voltage Switching (ZVS) is achieved for all semiconductors and, therefore, the SST can be operated at $48 \mathrm{kHz}$. More details about this SST can be found in [10], [51]. The MV/MF transformer, which is operated inside the DC-DC converter, is subject to all identified critical aspects with respect to dielectric losses: high-frequency, high switching speed, large common-mode (CM) voltages, and large differential-mode (DM) voltages.

Figs. 10(b)-(c) show the constructed transformer and Tab. I summarizes the key parameters. The stray inductance of the transformer, together with the series capacitor located on the LV side, is used as a resonant tank [5], [23]. The DC-DC converter is operated at the resonance frequency
TABLE I

PARAmeters of THE MV/MF TRANSFORMER

\begin{tabular}{ll}
\hline Parameter & Value \\
\hline Topology & Series-resonant DC-DC converter \\
Power & $25.0 \mathrm{~kW} / 13.6 \mathrm{kVAr}$ \\
Voltage & $\pm 3.5 \mathrm{kV}(\mathrm{DM}) / \pm 400 \mathrm{~V}(\mathrm{DM})$ \\
Current & $8.7 \mathrm{~A}(\mathrm{RMS}) / 73.0 \mathrm{~A}(\mathrm{RMS})$ \\
Frequency & $48.0 \mathrm{kHz}$ (switching) / 48.0 kHz (resonance) \\
Rise time & $580 \mathrm{~ns}$ (MV side) / 80 ns (LV side) \\
\hline Circuit & $L_{\sigma, \mathrm{MV}}=185 \mu \mathrm{H} / L_{\mathrm{m}, \mathrm{MV}}=4.6 \mathrm{mH}$ \\
\hline Winding & $52: 6 \mathrm{turns} /$ litz wire / shell-type \\
MV wire & $630 \times 71 \mu \mathrm{m} /$ three layers / two chambers \\
LV wire & $2500 \times 100 \mu \mathrm{m} /$ single layer \\
\hline Core type & Ferrite “BFM" / U-core / 2500 mm ${ }^{2}$ \\
Air gap & $2 \times 1.1 \mathrm{~mm}$ \\
\hline Cooling & Air-forced cooling / $40^{\circ} \mathrm{C}$ ambient \\
Insulation & Dry-type (silicone or epoxy) / vacuum potting \\
CM insulation & $4.0 \mathrm{~mm}$ thickness / designed for $15.0 \mathrm{kV}$ \\
\hline Box volume & $2.6 \mathrm{dm}{ }^{3}$ (transformer) \\
Box volume & $3.4 \mathrm{dm}{ }^{3}$ (transformer, fan, and terminations) \\
Mass & $6.2 \mathrm{~kg}$ (transformer, fan, and terminations) \\
Efficiency & $99.69 \% @ 25.0 \mathrm{~kW}$ (simulated) \\
Efficiency & $99.65 \pm 0.07 \%$ @ $25.0 \mathrm{~kW}$ (measured) \\
\hline
\end{tabular}
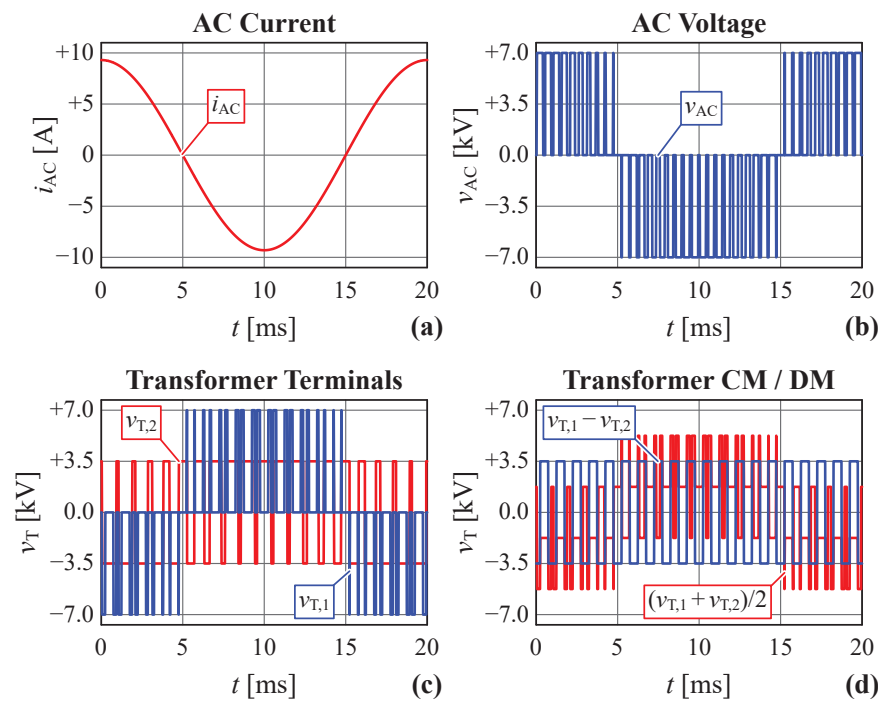

Fig. 12. Currents and voltages created by the MV side of the SST: (a) AC grid current, (b) AC PWM voltage, (c) MV/MF transformer terminal voltages, and (d) MV/MF transformer CM and DM voltages. For illustration purposes, a switching frequency of $1 \mathrm{kHz}$ is selected for the AC-DC and DC-DC stages (instead of $48 \mathrm{kHz}$ ).

and ZVS is achieved with the magnetizing current of the transformer [10]. This implies that the switched current is nearly load-independent and that the switching transitions feature a limited speed, which is beneficial for the dielectric losses [9], [23]. Due to the split DC-link half-bridge configuration, the DM voltage applied to the MV winding is $\pm 3.5 \mathrm{kV}$. Fig. 11 shows the measured voltages applied to the transformer and the corresponding currents [10].

In addition to the DM voltage applied to the transformer by the DC-DC stage, the AC-DC stage is creating an extra $\mathrm{CM}$ voltage. Fig. 12 illustrates the total voltages applied to the MV side of the transformer, given that system is 


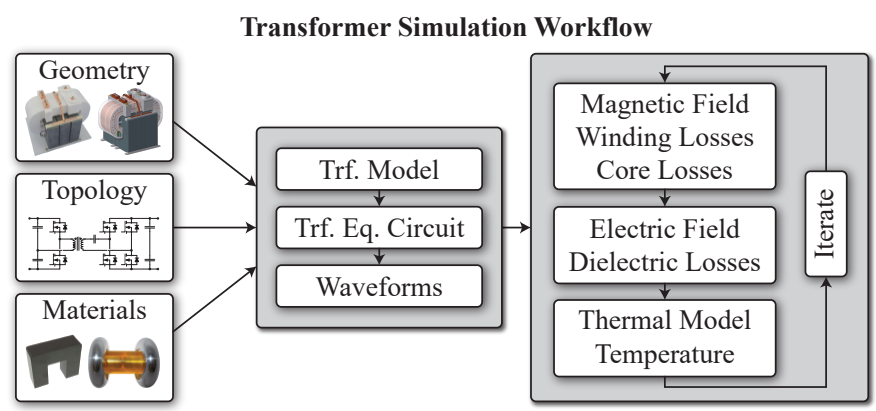

Fig. 13. Workflow for the simulation of the losses (core, winding, and insulation) of the MV/MF transformer. The electric field, magnetic field, and temperature distribution are obtained with FEM simulations [54].

grounded with respect to the AC grid (solid-earthing) on the MV side and with respect to the DC-link negative rail on the LV side. The first component of the CM voltage is generated by the AC-DC stage, i.e. the negative rail of the MV DC-link is jumping between $-7 \mathrm{kV}$ and $0 \mathrm{kV}$ with respect to the earth (at $48 \mathrm{kHz}$, with a sinusoidal modulation). The second component of the CM voltage is created by the DClink half-bridge configuration of the DC-DC stage, which is adding a constant $\mathrm{CM}$ voltage of $3.5 \mathrm{kV}$ [15]. The total $\mathrm{CM}$ voltage has an average value of $0 \mathrm{kV}$ over a complete grid period [15]. Altogether, a CM LF AC voltage, a CM MF PWM voltage, and a DM MF PWM voltage are applied to the transformer insulation and the peak voltage is $7 \mathrm{kV}$.

The transformer has been designed following the guidelines proposed in [12], [23], [52] and a ferrite core and litz wire windings have been chosen. A U-core based transformer allows for an efficient cooling of the winding near the winding head. A shell-type winding arrangement reduces the stray magnetic field in the winding window and, therefore, the high-frequency winding losses. The LV winding is placed near the core leg for several reasons: this winding arrangement improves the cooling of the MV winding, which is critical [14] and facilitates the realization of the MV cable terminations. Two air ducts are placed between the core and the winding package to allow for an efficient air-forced cooling (with a fan) [11], [12]. The MV winding is divided into two chambers and three layers which represents a good trade-off between the insulation stresses, the winding capacitance, and the complexity of the winding scheme [51], [53]. The transformer core is grounded with respect to the $\mathrm{LV}$ side such that no MV insulation is required for the LV winding. More details about the transformer design and construction can be found in [10], [23].

\section{B. Transformer Simulation}

Fig. 13 shows the workflow used for computing the transformer losses. The magnetic field and the electric field are computed with frequency domain FEM simulations, where the exact winding structure is considered (placement of the turns and layers) [54]. The temperature distribution $\left(40^{\circ} \mathrm{C}\right.$ ambient) is extracted with stationary FEM simulations [54]. Inside the transformer, only heat conduction processes are present (potted winding package with a temperatureindependent thermal conductivity). The heat extraction from
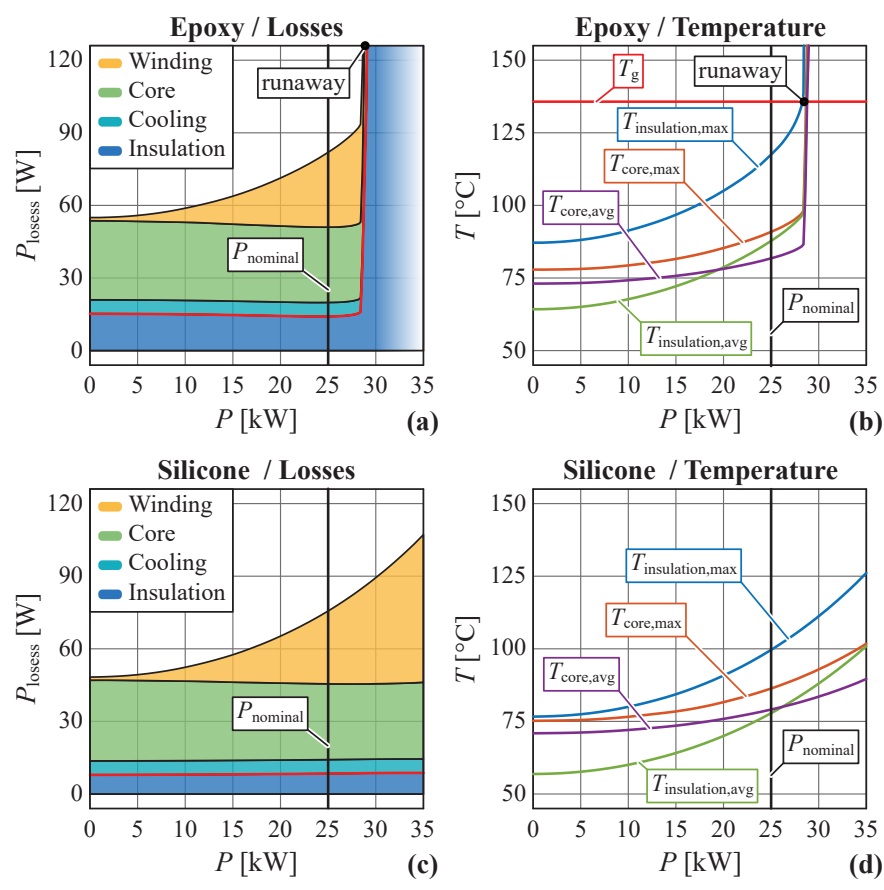

Fig. 14. (a) Simulated loss breakdown and (b) temperatures (averaged and hot spot) for the MV/MF transformer with an epoxy resin insulation ("vonRoll Damisol 3418"). (c) Simulated loss breakdown and (d) temperature (averaged and hot spot) for the MV/MF transformer with a silicone elastomer insulation ("Dow Corning TC4605 HLV"). For the epoxy resin, the glass transition temperature $\left(T_{\mathrm{g}}=136^{\circ} \mathrm{C}\right)$ and the associated thermal runaway are also indicated. The ambient temperature is set to $40^{\circ} \mathrm{C}$

the winding package and the core to the ambient is done with a forced convection process and the characteristic curve (pressure flow diagram) of the considered fan is integrated.

The spatial dependence of the losses and the frequency and temperature dependences of the material parameters are considered (i.e. non-uniform loss distribution). For each point (mesh element), the losses are computed with the applied stress (magnetic flux, electric field, or current density) and temperature. The consideration of non-uniform loss distribution is required for modeling eventual thermal runaways, which are occurring locally, at the temperature hotspots. An iterative process is used in order to find the equilibrium temperature distribution with the corresponding local values of the material parameters.

The winding losses (skin and proximity effects) are computed according to [12], the core losses (improved generalized Steinmetz equation with measured Steinmetz parameters) according to [55], and the dielectric losses (smallsignal frequency domain measurements of the complex permittivity with disc-shaped specimens) according to (9).

\section{Epoxy Resin Insulation}

In a first step, the transformer is insulated with the aforementioned "vonRoll Damisol 3418" unfilled epoxy resin (cf. Fig. 7) [49]. Figs. 14(a)-(b) show the simulated losses and the corresponding temperature distribution for different load conditions. As expected, the voltage related losses (core, cooling, and insulation) are approximately load-independent. Only the winding losses, which are related to the current, are (quadratically) load-dependent. 
TABLE II

Electric Field AND Dielectric Losses

\begin{tabular}{lcl}
\hline Parameter & Epoxy & Silicone \\
\hline Maximum electric field inside the insulation & \\
RMS field & $14.9 \mathrm{kV} / \mathrm{cm}$ & $15.0 \mathrm{kV} / \mathrm{cm}$ \\
Peak field & $22.4 \mathrm{kV} / \mathrm{cm}$ & $22.6 \mathrm{kV} / \mathrm{cm}$ \\
\hline Maximum electric field at the surface of the insulation \\
RMS field & $12.4 \mathrm{kV} / \mathrm{cm}$ & $12.5 \mathrm{kV} / \mathrm{cm}$ \\
Peak field & $18.6 \mathrm{kV} / \mathrm{cm}$ & $18.7 \mathrm{kV} / \mathrm{cm}$ \\
\hline Capacitive reactive power and dielectric losses \\
Capacitive reactive power & $1.0 \mathrm{kVar}$ & $1.2 \mathrm{kVar}$ \\
Total dielectric losses & $14.1 \mathrm{~W}$ & $8.4 \mathrm{~W}$ \\
Max. dielectric loss density & $405 \mathrm{~W} / \mathrm{m}^{3}$ & $273 \mathrm{~W} / \mathrm{m}^{3}$ \\
\hline \multicolumn{4}{c}{}
\end{tabular}

The key parameters (electric field and dielectric losses) are summarized in Tab. II at the nominal load $(25 \mathrm{~kW})$. The maximum peak electric field in the insulation is $22.6 \mathrm{kV} / \mathrm{cm}$, which is low for a dry-type insulation material [17], [56], [57]. However, the total capacitive reactive power, computed as the sum of the reactive power of the different harmonics, is $1.0 \mathrm{kVar}$. This implies that, even with a low dissipation factor, the dielectric losses represent $27 \%$ of the transformer losses at no-load operation and $17 \%$ at the nominal load.

For loads exceeding $28 \mathrm{~kW}$ ( $12 \%$ overload), a thermal runaway occurs in the insulation (inside the core window, between the windings). The mechanism of the thermal runaway can be explained as follows. The hot spot temperature of the transformer increases when evaluated over the load. This temperature increase is mainly produced by the winding losses which are strongly load-dependent. When the hot spot temperature reaches the glass transition temperature of the insulation material $\left(T_{\mathrm{g}}=136^{\circ} \mathrm{C}\right.$ for the considered epoxy resin), a massive increase of the dielectric losses occurs ( $\alpha$ peak, cf. Fig. 7). According to these results, the considered epoxy resin, which is a typical resin employed for LF systems, is less suitable at MF.

The considered unfilled resin features low-losses (cf. Fig. 7) and a high glass transition temperature $\left(T_{\mathrm{g}}=136^{\circ} \mathrm{C}\right)$ at the cost of a low thermal conductivity $(0.3 \mathrm{~W} / \mathrm{mK})$. Unfortunately, this problem is likely to appear with most epoxy resins. Filled epoxy resins will typically feature higher thermal conductivities but also higher losses and lower glass transition temperatures [34], [43]. Hence, the transformer prototype has not been insulated with epoxy resin.

\section{Silicone Elastomer Insulation}

Materials with significantly lower or higher glass transition temperatures (compared to the operating temperature) would be better suited for the insulation of MF transformers. Therefore, silicone elastomers which feature a low glasstransition temperature (below $0^{\circ} \mathrm{C}$ in the relevant frequency range) are considered, such that the loss peak associated with the glass transition temperature is not critical [58]. Furthermore the $\beta$ relaxation, which is much smaller than the $\alpha$ relaxation is occurring in the $\mathrm{MHz}$ range, i.e. above the corner frequency defined by the switching speed (cf. (16)). Therefore, over the complete operating range, no resonance peaks are encountered (cf. Fig. 3). The relatively high cost
TABLE III

Properties of the “Dow CORNING TC4605 HLV” Silicone

\begin{tabular}{ll}
\hline Property & Value \\
\hline Thermal conductivity & $1.0 \mathrm{~W} / \mathrm{mK}$ \\
Maximum temperature & $200^{\circ} \mathrm{C}$ \\
\hline Electrical resistivity & $10^{13} \Omega \mathrm{m}$ \\
Dielectric strength & $24 \mathrm{kV} / \mathrm{mm}$ \\
\hline Real permittivity & $\varepsilon^{\prime}<4.1\left(f>50 \mathrm{kHz} \wedge T<120^{\circ} \mathrm{C}\right)$ \\
Imaginary permittivity & $\varepsilon^{\prime \prime}<0.033\left(f>50 \mathrm{kHz} \wedge T<120^{\circ} \mathrm{C}\right)$ \\
Dissipation factor & $\tan \delta<0.8 \%\left(f>50 \mathrm{kHz} \wedge T<120^{\circ} \mathrm{C}\right)$ \\
\hline
\end{tabular}

of silicones (as compared to epoxies) is not a disqualifier for MF applications since the insulation volume is relatively small due to the high power densities.

For the considered transformer, the silicone elastomer "Dow Corning TC4605 HLV" has been chosen [59]. This elastomer features a relatively high breakdown strength, moderate permittivity, low dielectric losses, high thermal conductivity (the silicone is mixed with a filler), high temperature stability, low flammability, low viscosity (before curing), good mechanical stability (after curing), and good surface adhesion. The key properties of the material are summarized in Tab. III.

Figs. 14(c)-(d) show the simulated losses and the corresponding temperature distribution. The key parameters (electric field and dielectric losses) are summarized in Tab. II at the nominal load $(25 \mathrm{~kW})$. Compared to the epoxy insulation, the maximum RMS electric field, maximum peak electric field, and the capacitive reactive power are similar. However, the dielectric losses are lower: $16 \%$ of the transformer losses at no-load operation and $11 \%$ at the nominal load. The simulation shows that with silicone insulation, the transformer could be operated at $35 \mathrm{~kW}(40 \%$ overload) without experiencing excessive temperature or a thermal runaway.

Fig. 15 depicts the RMS electric field and the insulation losses. Due to the split DC-link half-bridge configuration, the electric field pattern is slightly asymmetric between the two halves of the core sets (cd. Fig. 12) [15]. As expected, the electric field is most pronounced between the MV and LV windings and inside the MV winding. Due to the permittivity mismatch between the silicone and the air, the electric field in the air ducts is particularly large, but below the ionization electric field in air [17], [23]. Since the insulation losses are proportional to the square of the electric field, the dielectric losses are only significant near the surface of the MV winding.

\section{E. Dielectric Loss Measurements}

From the aforementioned considerations and simulations, the silicone elastomer "Dow Corning TC4605 HLV" appears to be superior to epoxy resins and, therefore, has been chosen for insulating the transformer prototype. The LV and MV windings are wound on a polycarbonate coil former, potted with a vacuum potting process ( $30 \mathrm{mbar})$, and cured in an oven $\left(120^{\circ} \mathrm{C}\right)$.

Due to the low dissipation factor, dielectric losses are only accounting for a small part of the total transformer 

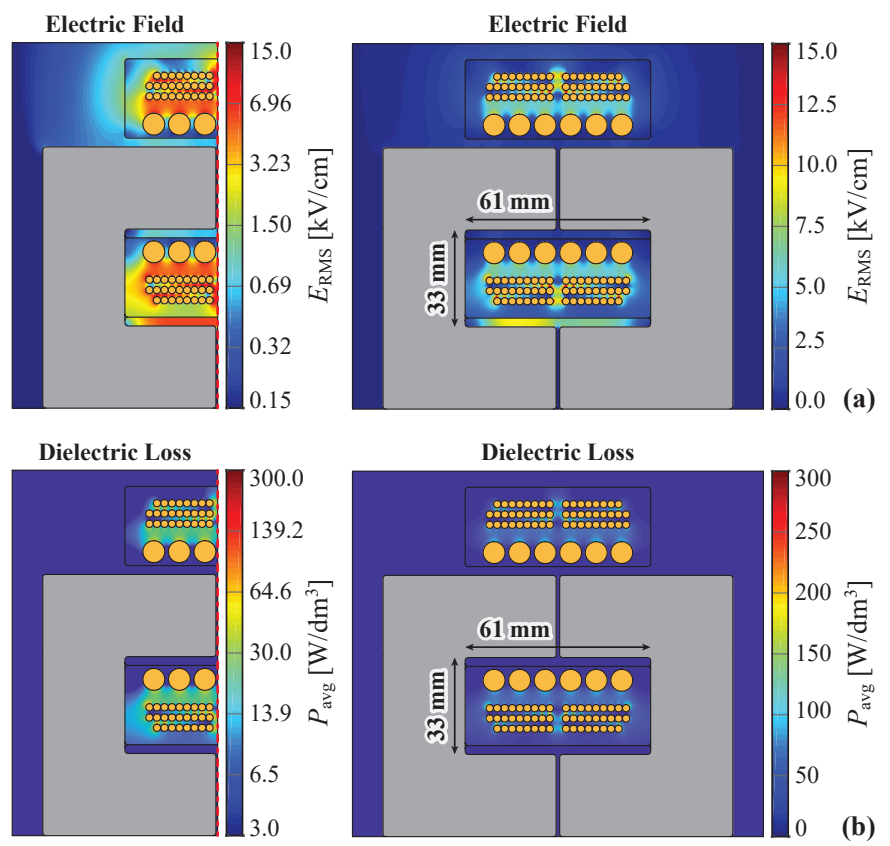

(a)

Fig. 15. (a) RMS value of the electric field and (b) dielectric loss density in the insulation (logarithmic and linear scales). The simulations are conducted at rated conditions $(25 \mathrm{~kW})$ with the silicone elastomer "Dow Corning TC4605 HLV" as insulation material. Due to the permittivity mismatch between the silicone and the air, the electric field is pushed towards the air ducts, given that the magnetic core is grounded.

losses, i.e. $16 \%$ at no-load operation and $11 \%$ at the nominal load. Therefore, the extraction of the dielectric losses during rated operation is intrinsically ill-conditioned and, therefore, inaccurate. For this reason, the measurement of the insulation losses is done with pure CM excitations between the MV and LV windings (the core is grounded with respect to the LV side). With such an excitation, no core or winding losses are occurring and the presented dielectric loss computation methods can be validated. The following excitations are considered:

- $\mathrm{S}_{50 \mathrm{k}}$ - A MV/MF sinusoidal voltage with a frequency of $50 \mathrm{kHz}$ and a peak amplitude of $\pm 3.6 \mathrm{kV}$.

- $\mathrm{S}_{100 \mathrm{k}}$ - A MV/MF sinusoidal voltage with a frequency of $100 \mathrm{kHz}$ and a peak amplitude of $\pm 3.6 \mathrm{kV}$.

- $\mathrm{P}_{890 \mathrm{n}}$ - A MV/MF PWM voltage with a switching frequency of $50 \mathrm{kHz}$, a duty cycle of $50 \%$, a rise time $(10 \%-90 \%)$ of $890 \mathrm{~ns}$, and an amplitude of $\pm 3.5 \mathrm{kV}$.

- $\mathrm{P}_{480 \mathrm{n}}$ - A MV/MF PWM voltage with a switching frequency of $50 \mathrm{kHz}$, a duty cycle of $50 \%$, a rise time $(10 \%-90 \%)$ of $480 \mathrm{~ns}$, and an amplitude of $\pm 3.5 \mathrm{kV}$.

Fig. 16 shows the measured voltages and the associated $\mathrm{CM}$ current through the insulation $\left(C_{\mathrm{CM}}=139.3 \mathrm{pF}\right)$. The sinusoidal voltages are generated with a series-resonant circuit fed by a linear amplifier. The PWM voltages are generated by a $10 \mathrm{kV}$ SiC MOSFETs bridge [9], [10]. The switching speed of the MOSFETs is adjusted with the ZVS current, which is provided by a parallel inductor featuring an adjustable air-gap.

The electrical measurement of the dielectric losses is difficult since most dielectric measurement systems are working with small-signal sinusoidal waveforms and cannot deal
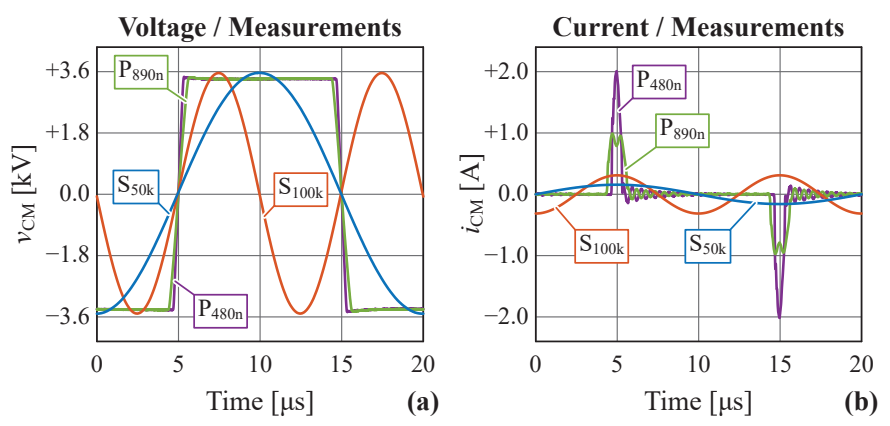

Fig. 16. (a) Measured CM voltages applied between the MV winding and the LV winding (the core is grounded with respect to the LV side). (b) CM charging current flowing through the MV winding.

with MV/MF sinusoidal or MV/MF PWM voltages. Therefore, the following measurement methods are considered (at an ambient temperature of $22^{\circ} \mathrm{C}$ ):

- Meas. / LV frequency - The transformer is measured with small signal excitation and the spectral components of the dielectric losses are extracted. The low-frequency measurement (below $150 \mathrm{kHz}$ ) are done with the setup described in [36], [37] and the high-frequency measurements (above $150 \mathrm{kHz}$ ) are obtained with a precision impedance analyzer [60]. Both measurement setups are carefully calibrated with a quasi-lossless gas reference capacitor [61]. Afterwards, the losses associated with the aforementioned excitations are reconstructed using (10) with the hypothesis that the dielectric polarization of the insulation material behaves linearly with respect to the electric field. The peak voltage applied to the insulation varies, depending on the used setup, between $1.4 \mathrm{~V}$ and $140 \mathrm{~V}$. Since the dielectric losses are proportional to the square of the voltage, this implies that the measured losses are between $10^{2}$ and $10^{7}$ times smaller than the unscaled values.

- Meas. / MV calorimetric - The transformer is measured with the MV signals and the losses are extracted with transient calorimetric measurements [9]. The windings are equipped with four NTC thermistors (two at the surface of the potted windings and two inside the windings) and the complete transformer (without the fan) is brought in a thermally insulated polystyrene box. In a first step, the relations between the measured temperatures and the losses are extracted by injecting DC currents in the MV winding (between $1 \mathrm{~W}$ and $10 \mathrm{~W}$ of losses). Afterwards, the transformer is fed with the MV CM voltages (cf. Fig. 16) and the temperatures are recorded. Then, it is straightforward to extract the dielectric losses from the calibration data. The duration of the calibrations and measurements is set to $18 \mathrm{~min}$ and the time constant of the system is $35 \mathrm{~min}$ (without the fan). This implies that the measurement is done during the temperature transient. The temperature elevation remains under $3^{\circ} \mathrm{C}$, implying that the temperature dependence of the permittivity can be neglected. This measurement method does not rely on the linearity of the insulation material. The only hypothesis is that the temperature distribution is 
TABLE IV

Measured and Simulated Transformer CM Losses

\begin{tabular}{lllll}
\hline Meas. / Sim. Method & $\mathbf{S}_{\mathbf{5 0 k}}$ & $\mathbf{S}_{\mathbf{1 0 0 k}}$ & $\mathbf{P}_{\mathbf{8 9 0 n}}$ & $\mathbf{P}_{\mathbf{4 8 0 n}}$ \\
\hline Meas. / LV Frequency & $1.30 \mathrm{~W}$ & $2.35 \mathrm{~W}$ & $3.05 \mathrm{~W}$ & $3.63 \mathrm{~W}$ \\
Meas. / MV Calorimetric & $1.23 \mathrm{~W}$ & $2.49 \mathrm{~W}$ & $3.02 \mathrm{~W}$ & $3.98 \mathrm{~W}$ \\
\hline Sim. / Infinite Summation & $1.33 \mathrm{~W}$ & $2.54 \mathrm{~W}$ & $3.37 \mathrm{~W}$ & $4.07 \mathrm{~W}$ \\
Sim. / Imag. Eps. Approx. & $\mathrm{n} / \mathrm{a}$ & $\mathrm{n} / \mathrm{a}$ & $3.36 \mathrm{~W}$ & $3.91 \mathrm{~W}$ \\
Sim. / Real Eps. Approx. & $\mathrm{n} / \mathrm{a}$ & $\mathrm{n} / \mathrm{a}$ & $3.26 \mathrm{~W}$ & $3.78 \mathrm{~W}$ \\
\hline
\end{tabular}

similar during calibrations and measurements, which is confirmed by the comparison of the measured time constants and temperatures.

The computation of the losses is done with the CM electric field obtained from FEM $\left(C_{\mathrm{CM}}=149.8 \mathrm{pF}\right)$ [54]. The insulation material properties are obtained with small-signal frequency domain measurements of the complex permittivity on a disc-shaped specimen. Then the following methods are used for computing the losses (cf. Section III):

- Sim. / Infinite Summation - The dielectric losses are obtained with an infinite summation over the harmonics (cf. (10)). For the sinusoidal voltages, only one term is non-zero.

- Sim. / Imag. Eps. Approx. - The approximations based on the imaginary part of the permittivity are used for extracting the losses (cf. (27), (28), (29), and (30)). This method is only applicable to PWM voltages (not for sinusoidal).

- Sim. / Real Eps. Approx. - The approximations based on the real part of the permittivity are used for extracting the losses (cf. (31), (32), (33), (34)). This method is only applicable to PWM voltages (not for sinusoidal).

Tab. IV depicts the calculated and measured losses. The maximum error between the two measurement methods is below $9 \%$ and the maximum error between the measurements and the computations is below $13 \%$. These deviations matches with the estimated measurement uncertainties. For the MV calorimetric measurements, the total estimated uncertainty is $16 \%$, where the temperature measurement (6\%, datasheet of the NTC thermistors and ADCs) and the error due to the discrepancies between the calibration and the measurements ( $10 \%$, estimated with thermal FEM simulations) are considered. Hence, the following hypotheses are verified by the aforementioned measurements:

- The dielectric response is linear with the electric field and, therefore, the losses of the different harmonics can be measured with small signal excitations and combined. This point is proven by comparing the small and large signal measurements (both for sinusoidal and PWM voltages).

- The electric field pattern computed with FEM is sufficiently accurate for computing the losses. This is verified by comparing the measured CM capacitance and losses to the simulated values. This implies that the dielectric loss simulations done for the transformer during rated conditions (with CM and DM voltages, cf. Subsection IV-B) are valid.

- The proposed approximations (cf. Subsection III-D) can be used to avoid the summation of the spectral losses.
This greatly facilitates the computation of the dielectric losses from FEM simulations since no computationally intensive summations on FEM meshes are required.

\section{Design Guidelines}

The presented approaches and results can be combined in order to extract design guidelines for choosing appropriate MV/MF (dry-type) insulation materials and computing the corresponding dielectric losses:

- First, the relevant frequency and temperature ranges should be identified for the insulation system. The frequency range spans from the switching frequency to the corner frequency defined by the switching speed of the semiconductors (cf. (16)). The obtained switching speed depends on the used semiconductors and the switching conditions (e.g., ZVS and hardswitching). The usage of converter topologies featuring multiple levels (e.g., cascaded bridges, modular multilevel converter) [15], [25] and/or ZVS (e.g., dual active bridge, series resonant converter) [2], [5], [8], [10] reduces the stress applied to the insulation due to the smaller voltage steps and the reduced switching speeds, respectively.

- The insulation material should be chosen such that no large loss peak occurs in the aforementioned operating range (cf. Fig. 3). Particularly, the $\alpha$ relaxation (glass transition temperature) puts a constraint on the temperature range. The $\beta$ relaxation is less critical but the associated superlinear increase of the dielectric losses as a function of operating frequency should be taken into account. For most insulation materials, the permittivity and dissipation factor provided in the datasheets do not cover the required frequency and temperature ranges. Depending on the required accuracy, the missing information can be extrapolated, taken from available material databases, or measured with a dielectric spectroscopy setup [37], [43], [48].

- If the permittivity curve is almost constant over the considered spectrum (absence of loss peaks), the approximation (20) applies. If the frequency dependence of the permittivity is moderate (absence of loss peaks), the approximations (27) and (31) can be used. For other cases, the computationally intensive summation (10) is required.

- In power converter insulations, the dielectric materials are usually not the primary source of losses (e.g., core, winding, and semiconductors). If the external losses (e.g., winding losses and core losses) are dominating, the dielectric losses can be neglected for computing the temperature distribution. If the external losses and the dielectric losses are in the same range, the impact of the dielectric losses on the temperature distribution should be taken into account with an iterative process. This iterative process is also required for detecting thermal runaways.

- The dielectric losses with MV excitations can be measured with calorimetric methods. However, due to the linearity of dry-type polymeric insulation materials, small-signal frequency domain spectroscopy can also be used. Such measurements can be conducted with 
complete prototypes or material samples. The latter is particularly useful during design phases. Moreover, some capacitances (e.g., DM capacitance of transformers) cannot be measured with complete prototypes without exciting non-dielectric effects (e.g., conduction losses and magnetic losses).

The measurements conducted on the MV/MF transformer prototype prove that the dielectric losses are a critical parameter for MV/MF insulation. However, the choice of a $\mathrm{MV} / \mathrm{MF}$ insulation material cannot be reduced to the sole dielectric losses. Many other factors influence the suitability of a material for a specific application:

- High Voltage - The breakdown voltage, the bulk conductivity, and the resistance against partial discharges are important properties for MV/MF insulation materials [20]-[22], [30], [31]. The DC conductivity is particularly important for determining the DC electric field distribution [23], [38], [46].

- Permittivity - Even if the dielectric losses are proportional to the imaginary part of the permittivity (cf. (10)), the real part is also an important design parameter since it is responsible for the capacitive reactive power in the insulation. Relatively large displacement currents may create losses in the grounding system, oscillations, and EMI issues [6], [23].

- Thermal - The thermal conductivity of the insulation is critical since it directly defines the thermal performance of the system. However, materials with high thermal conductivity often feature poor (di)electric performance [43], [46]. Furthermore, the maximum operating temperature (thermal aging) and the flammability of the material should be taken into account.

- Chemical - The resistance to moisture and corrosion, as well as the chemical compatibility with other materials (e.g., glue, windings, and coil formers), has to be considered. The aging of the material is also an important issue [20], [22].

- Mechanical - The mechanical properties (e.g., flexible, rigid, brittle, surface adhesion, and thermal expansion), as well as the processing capabilities (e.g., milling, potting, viscosity, and presence of cavities or defects), should also be investigated [46].

Trade-offs are required between these properties. The unfilled epoxy resin ("vonRoll Damisol 3418") considered in this work features a high glass transition temperature, relatively low losses, a moderate permittivity, and a good mechanical stability. However, this material has a low thermal conductivity, is brittle, and exhibits high losses near the glass transition temperature. An unfilled silicone elastomer, compared to the selected filled silicone elastomer ("Dow Corning TC4605 HLV"), would have lower losses but also lower thermal conductivity and a reduced mechanical stability [58]. Generally, the adjunction of a thermally conductive filler (e.g., $\mathrm{SiO}_{2}$ and $\mathrm{Al}_{2} \mathrm{O}_{3}$ ) increases the permittivity, degrades the (di)electric performance, and increases the viscosity of the polymer (before curing).

Non-pottable and/or non-polymeric insulation materials, such as mica, ceramic (e.g., AlN and AlSiC), aramid (e.g., Nomex), polyimide (e.g., Kapton) also feature interesting properties, i.e. high breakdown strength (especially for thin layers), good resistance to partial discharges, and low losses [43], [62]. These materials are typically combined with potted polymeric materials or oils, in order to avoid the presence of air near the electric field hot spots, which can lead to partial discharges [56].

\section{CONCLUSION}

This paper examines the dielectric losses in the insulation of MV converters. The dielectric losses are a quantitative indicator for the MF insulation stress, which is useful for design and diagnostic processes. First, the modeling of dielectric losses in time and frequency domain is reviewed. Afterwards, the impact of PWM voltages generated by typical power converters on the dielectric losses is studied. Analytical expressions for calculating the dielectric losses of the frequency-dependent materials used in dry-type insulation systems are derived. It is shown that fundamental frequency analysis inaccurately determines the dielectric losses with PWM voltages. For obtaining the correct spectrum, a model of the switching transitions is also required. The proposed approximations are verified with permittivity measurements conducted for a typical MV insulation epoxy resin at different frequencies and temperatures (less than $16 \%$ error). It is shown that, for accurate loss predictions, the frequency and temperature dependences of the material parameters should be considered.

The described computation techniques are applied to a $\mathrm{MV} / \mathrm{MF}$ transformer used in the DC-DC converter $(7 \mathrm{kV}$ to $400 \mathrm{~V}, 48 \mathrm{kHz}$, and $25 \mathrm{~kW}$ ) of a SST. With the considered epoxy resin, the insulation losses reach $17 \%$ of the total transformer losses. Moreover, a thermal runaway, associated with the loss peak at the glass transition temperature, limits the achievable power rating. The usage of a high performance silicone elastomer mitigates the aforementioned problems. The realized prototype, which features a silicone elastomer insulation, is measured with small-signal spectroscopy and with MV large-signal calorimetric methods. It is shown that the deviations between the measurements and the proposed computation methods are below $13 \%$. Finally, guidelines are given for selecting suitable insulation materials for MV/MF applications.

\section{APPENDIX}

\section{A. PWM with Frequency-Independent Materials}

This is the derivation of the approximation of the dielectric losses produced by frequency-independent materials under PWM voltages (cf. (20), (21), and (22)). From (18) and (19), the normalized losses $P^{\prime}$ can be written as (with the hypothesis $\varepsilon_{\mathrm{r}}^{\prime \prime}(f)=$ const.):

$$
P^{\prime}=\frac{P}{\varepsilon_{\mathrm{r}}^{\prime \prime} C_{0} V_{\mathrm{DC}}^{2}}=\sum_{n=1}^{\infty} \frac{4 f_{\mathrm{s}}}{\pi} \frac{\sin ^{2}\left(\pi n D_{\mathrm{c}}\right)}{n} \frac{1}{1+\left(n \frac{f_{\mathrm{s}}}{f_{\mathrm{c}}}\right)^{2}} .
$$

The infinite summation can be approximated by a finite summation (until the corner frequency of the first-order low-pass filter, cf. (16)). The following expression is derived (using the double angle trigonometric formula):

$$
P^{\prime} \approx \sum_{n=1}^{\left\lceil f_{\mathrm{c}} / f_{\mathrm{s}}\right\rceil} \frac{4 f_{\mathrm{s}}}{\pi} \frac{1-\cos \left(2 \pi D_{\mathrm{c}} n\right)}{2 n},
$$


which can be rewritten as

$$
P^{\prime} \approx \frac{2 f_{\mathrm{s}}}{\pi}\left(\sum_{n=1}^{\left\lceil f_{\mathrm{c}} / f_{\mathrm{s}}\right\rceil} \frac{1}{n}-\sum_{n=1}^{\left\lceil f_{\mathrm{c}} / f_{\mathrm{s}}\right\rceil} \frac{(-1)^{n} \cos \left(2 \pi n D_{\mathrm{c}}-n \pi\right)}{n}\right) .
$$

An approximation exists for the partial sum of the first term, which is a harmonic series [47]. The second term converges quickly such that the finite sum can be approximated with the infinite sum, for which a closed-form solution exists [63]. This leads to

$$
P^{\prime} \approx \frac{2 f_{\mathrm{s}}}{\pi}\left(\ln \left(\frac{f_{\mathrm{c}}}{f_{\mathrm{s}}}\right)+\gamma+\frac{1}{2} \ln \left(2+2 \cos \left(2 \pi D_{\mathrm{c}}-\pi\right)\right)\right) .
$$

This last expression can be further simplified with the double angle trigonometric formula:

$$
P^{\prime} \approx \frac{2 f_{\mathrm{s}}}{\pi} \ln \left(2 \mathrm{e}^{\gamma} \frac{f_{\mathrm{c}}}{f_{\mathrm{s}}} \sin \left(\pi D_{\mathrm{c}}\right)\right),
$$

which is equivalent, after some trivial algebraic operations, to the expressions proposed in (20), (21), and (22).

\section{B. Upper Bound on the Dielectric Losses}

This is the derivation of the proposed expression for the losses produced by frequency-dependent materials subject to a single (non-periodic) switching transition (cf. (23) and (25)). The transition is modeled as the step response of a first-order low-pass filter, cf. Fig. 4(b)). First, the Fourier transform of the applied signal is considered:

$$
V(f)=\int_{0}^{\infty}\left(1-\mathrm{e}^{-2 \pi f_{\mathrm{c}} t}\right) \mathrm{e}^{-\mathrm{j} 2 \pi f t} \mathrm{~d} t .
$$

This Fourier transform features an analytical solution:

$$
V(f)=\frac{1}{2 \pi}\left(\frac{1}{\mathrm{j} f}+\frac{1}{\mathrm{j} f+\mathrm{j} f_{\mathrm{c}}}+\pi \delta(f)\right),
$$

where $\delta$ is the Dirac delta function. Then, the normalized losses can be computed from the integration of the spectral losses (Parseval's theorem):

$$
W^{\prime}=\frac{W}{C_{0} V_{\mathrm{DC}}^{2}}=2 \int_{0}^{\infty} \varepsilon_{\mathrm{r}}^{\prime \prime}(2 \pi f)|V(f)|^{2} \mathrm{~d} f,
$$

which can be rewritten as

$$
W^{\prime}=\frac{1}{\pi} \int_{0}^{\infty} \varepsilon_{\mathrm{r}}^{\prime \prime}(f) \frac{1}{f} \frac{f_{\mathrm{c}}^{2}}{f^{2}+f_{\mathrm{c}}^{2}} \mathrm{~d} f
$$

which is equivalent to the expression (23). This equation can be expanded as

$$
W^{\prime}=\frac{1}{2} \varepsilon_{\mathrm{r}}^{\prime}(0)-\frac{1}{2} \varepsilon_{\mathrm{r}}^{\prime}(0)+\frac{1}{\pi} \int_{0}^{\infty} \varepsilon_{\mathrm{r}}^{\prime \prime}(f) \frac{1}{f} \frac{f_{\mathrm{c}}^{2}}{f^{2}+f_{\mathrm{c}}^{2}} \mathrm{~d} f .
$$

This last expression can be transformed by introducing the Kramers-Kronig relation for the real part of the permittivity (cf. (6)). This leads to

$$
W^{\prime}=\frac{\varepsilon_{\mathrm{r}}^{\prime}(0)-\varepsilon_{\mathrm{r}}^{\prime}(\infty)}{2}+\frac{1}{\pi} \int_{0}^{\infty} \varepsilon_{\mathrm{r}}^{\prime \prime}(f) \frac{1}{f}\left(\frac{f_{\mathrm{c}}^{2}}{f^{2}+f_{\mathrm{c}}^{2}}-1\right) \mathrm{d} f,
$$

which is equivalent to the expression (25).
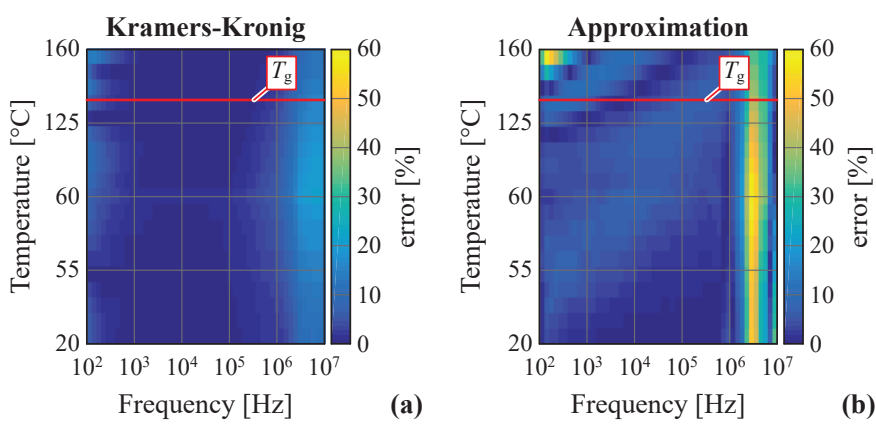

Fig. 17. Relative error between the measured $\varepsilon_{\mathrm{r}}^{\prime \prime}(f, T)$ and the values computed with (a) the formula (7) and (b) the approximation (8). The epoxy resin "vonRoll Damisol 3418" is considered and the glass transition temperature $\left(T_{\mathrm{g}}=136^{\circ} \mathrm{C}\right)$ is indicated. The measured complex permittivity is shown in Fig. 7 .

\section{PWM with Frequency-Dependent Materials}

This is the derivation of the approximation of the dielectric losses produced by frequency-dependent materials under PWM voltages (cf. (27), (28), (29), and (30)). First, the duty cycle is fixed to $D_{\mathrm{c}}=50 \%$. From (18) and (19), the normalized losses $P^{\prime}$ can be written as

$$
P^{\prime}=\frac{P}{C_{0} V_{\mathrm{DC}}^{2}}=\sum_{n=1}^{\infty} \frac{4 f_{\mathrm{s}}}{\pi} \frac{\varepsilon_{\mathrm{r}}^{\prime \prime}\left(n f_{\mathrm{s}}\right) \cdot \sin ^{2}\left(\frac{\pi}{2} n\right)}{n} \frac{1}{1+\left(n \frac{f_{\mathrm{s}}}{f_{\mathrm{c}}}\right)^{2}} .
$$

With the chosen duty cycle, only the odd harmonics are non-zero. As shown in Appendix A, the summation can be truncated at the corner frequency:

$$
P^{\prime} \approx \sum_{n=1 \text { (odd) }}^{\left\lceil f_{\mathrm{c}} / f_{\mathrm{s}}\right\rceil} \frac{4 f_{\mathrm{s}}}{\pi} \frac{\varepsilon_{\mathrm{r}}^{\prime \prime}\left(n f_{\mathrm{s}}\right)}{n} .
$$

This summation can be approximated with an integral [64]. The inaccuracy of this approximation near the fundamental frequency is compensated by an additional term $P_{\text {add }}^{\prime}$ :

$$
P^{\prime} \approx P_{\text {add }}^{\prime}+\frac{1}{2} \frac{4 f_{\mathrm{s}}}{\pi} \int_{\ln \left(f_{\mathrm{s}}\right)}^{\ln \left(f_{\mathrm{c}}\right)} \varepsilon_{\mathrm{r}}^{\prime \prime}(f) \mathrm{d}(\ln (f)),
$$

where the factor $1 / 2$ results from the fact that only odd values of $n$ are considered. The term $P_{\text {add }}^{\prime}$ is chosen such that the approximation is equivalent to (39) for the special case of a frequency-independent $\varepsilon_{\mathrm{r}}^{\prime \prime}(f)$. This leads to

$$
P_{\text {add }}^{\prime}=\frac{2 f_{\mathrm{s}}}{\pi} \ln \left(2 \mathrm{e}^{\gamma} \sin \left(\pi D_{\mathrm{c}}\right)\right) \varepsilon_{\mathrm{r}}^{\prime \prime}\left(f_{\mathrm{s}}\right),
$$

which, together with (48), is equal, after some trivial algebraic operations, to the expressions proposed in (27), (28), (29), and (30).

\section{Accuracies of the Kramers-Kronig Approximation}

The Kramers-Kronig relations (cf. (7)) are compared with the approximation (cf. (8)). For the comparison, the epoxy resin "vonRoll Damisol 3418", which presents the typical characteristics of a polymeric insulation material, is used (cf. Fig. 7) [49]. 
TABLE V

PWM WITH FREQUENCY-INDEPENDENT MATERIALS

\begin{tabular}{lll}
\hline Considered parameter combinations (cf. Fig. 4) \\
$f_{\mathrm{s}} \in[1,100] \mathrm{kHz} / t_{\mathrm{r}} \in[10,1000] \mathrm{ns} / D_{\mathrm{c}} \in[10 \%, 90 \%]$ \\
$t_{\mathrm{r}} f_{\mathrm{s}}<0.2 D_{\mathrm{c}} / t_{\mathrm{r}} f_{\mathrm{s}}<0.2\left(1-D_{\mathrm{c}}\right)$ \\
Reference values are obtained with (10) \\
\hline Equations & Low-pass & Ramp \\
$(21)$ & $78.8 \%$ & $78.6 \%$ \\
$(20) /(21) /(22)$ & $0.6 \%$ & $4.0 \%$ \\
\hline
\end{tabular}

TABLE VI

PWM with FreQuency-DePendent Materials

Considered parameter combinations (cf. Fig. 4)
$f_{\mathrm{s}} \in[1,100] \mathrm{kHz} / t_{\mathrm{r}} \in[100,1000] \mathrm{ns} / D_{\mathrm{c}} \in[10 \%, 90 \%]$
$t_{\mathrm{r}} f_{\mathrm{s}}<0.2 D_{\mathrm{c}} / t_{\mathrm{r}} f_{\mathrm{s}}<0.2\left(1-D_{\mathrm{c}}\right)$
$T \in[20,160]^{\circ} \mathrm{C} /$ "vonRoll Damisol 3418 " epoxy resin
\begin{tabular}{lll} 
Reference values are obtained with $(10)$ \\
\hline Equations & Low-pass & Ramp \\
$(21)$ & $90.4 \%$ & $90.0 \%$ \\
$(20) /(21) /(22)$ & $109.4 \%$ & $111.6 \%$ \\
$(27) /(28) /(29) /(30)$ & $7.1 \%$ & $7.6 \%$ \\
$(31) /(32) /(33) /(34)$ & $16.1 \%$ & $10.6 \%$ \\
\hline
\end{tabular}

Fig. 17(a) shows the relative error between the measured $\varepsilon_{\mathrm{r}}^{\prime \prime}(f, T)$ and the values computed with (7). Due to the presence of singularities, the numerical integration of (7) is challenging. In this work, the Maclaurin's formula has been used, as described in [65]. The error between the two curves can be explained by several factors [66]: the accuracy of the measurements, the presence of non-linearities (especially for reduced frequencies), and the fact that (7) requires the permittivity for the complete spectrum (from $0 \mathrm{~Hz}$ to $\infty \mathrm{Hz}$ ). Therefore, the computation is less accurate at the boundaries of the measured frequency range. Nevertheless, the deviation is below $17 \%$ for the complete range and below $7 \%$ for frequencies and temperatures located outside the relaxation peaks (below $1 \mathrm{MHz}$ and below $120^{\circ} \mathrm{C}$ ).

Fig. 17(b) depicts the relative error between the measured $\varepsilon_{\mathrm{r}}^{\prime \prime}(f, T)$ and the values computed with (8). The local approximation is accurate (less than $9 \%$ error) for frequencies and temperatures located outside the relaxation peaks (below $1 \mathrm{MHz}$ and below $120^{\circ} \mathrm{C}$ ). For the complete range, the maximum error reaches $60 \%$, indicating that the approximation of the Kramers-Kronig relations should not be used around the loss peaks.

\section{E. Accuracies of the Dielectric Losses Approximations}

The proposed approximations for the loss computation are compared to the summation (10). For the summation, two approximations of the switching transitions are used: the ramp function ("ramp") and the step response of a first-order low-pass filter ("low-pass"), cf. Fig. 4(b)). The considered parameter ranges are based on typical values used for MV converters. The obtained maximum relative errors over all the possible combinations are considered. A condition is set on the duty cycle, switching frequency, and switching speed in order to avoid quasi-triangular pulses.
Tab. V contains the results for the losses of frequencyindependent materials subject to PWM voltages (cf. Subsection III-A). It appears that the proposed approximation is valid. A fundamental frequency analysis of PWM voltages is invalid $(78 \%$ error).

Tab. VI shows the errors for the losses with frequency and temperature dependent materials subject to PWM voltages (cf. Subsection III-D). For the analysis, the epoxy resin "vonRoll Damisol 3418", which presents the typical characteristics of a polymeric insulation material, is considered (cf. Fig. 7) [49]. The proposed approximations are valid. It appears that the frequency dependence of the permittivity cannot be neglected (111\% error).

\section{ACKNOWLEDGMENT}

The research has been carried out within the frame of the Swiss Centre for Competence in Energy Research on the Future Swiss Electrical Infrastructure (SCCER-FURIES) with the financial support of the Swiss Innovation Agency.

\section{REFERENCES}

[1] D. Rothmund, G. Ortiz, T. Guillod, and J. W. Kolar, “10kV SiC-Based Isolated DC-DC Converter for Medium Voltage-Connected Solid-State Transformers," in Proc. of the IEEE Applied Power Electronics Conf. and Expo. (APEC), Mar. 2015.

[2] A. Tripathi, S. Madhusoodhanan, K. Mainali, K. Vechalapu et al., "Enabling DC Microgrids with Direct MV DC Interfacing DAB Converter based on $15 \mathrm{kV}$ SiC IGBT and $15 \mathrm{kV} \mathrm{SiC} \mathrm{MOSFET,"} \mathrm{in} \mathrm{Proc.} \mathrm{of} \mathrm{the}$ IEEE Energy Conversion Congr. and Expo. (ECCE USA), Sep. 2016.

[3] C. Zhao, D. Dujic, A. Mester, J. K. Steinke et al., "Power Electronic Traction Transformer - Medium Voltage Prototype," IEEE Trans. Ind. Electron., vol. 61, no. 7, pp. 3257-3268, 2014.

[4] D. Rothmund, G. Ortiz, and J. W. Kolar, "SiC-based Unidirectional Solid-State Transformer Concepts for Directly Interfacing 400V DC to Medium-Voltage AC Distribution Systems," in Proc. of the IEEE Telecommunications Energy Conf. (INTELEC), Sep. 2014.

[5] L. Wang, Q. Zhu, W. Yu, and A. Q. Huang, "A Medium-Voltage MediumFrequency Isolated DC-DC Converter Based on 15-kV SiC MOSFETs," IEEE J. Emerg. Sel. Topics Power Electron., vol. 5, no. 1, pp. 100-109, Mar. 2017.

[6] S. Madhusoodhanan, A. Tripathi, D. Patel, K. Mainali et al. "SolidState Transformer and MV Grid Tie Applications Enabled by $15 \mathrm{kV}$ SiC IGBTs and $10 \mathrm{kV}$ SiC MOSFETs Based Multilevel Converters," IEEE Trans. Ind. Appl., vol. 51, no. 4, pp. 3343-3360, 2015.

[7] A. Q. Huang, M. L. Crow, G. T. Heydt, J. P. Zheng, and S. J. Dale, "The Future Renewable Electric Energy Delivery and Management (FREEDM) System: The Energy Internet," Proc. IEEE, vol. 99, no. 1, pp. 133-148, 2011

[8] R. W. De Doncker, D. M. Divan, and M. H. Kheraluwala, "A Three-Phase Soft-Switched High-Power-Density DC/DC Converter for High-Power Applications," IEEE Trans. Ind. Appl., vol. 27, no. 1, pp. 63-73, 1991.

[9] D. Rothmund, D. Bortis, and J. W. Kolar, "Accurate Transient Calorimetric Measurement of Soft-Switching Losses of 10kV SiC MOSFETs and Diodes," IEEE Trans. Power Electron., vol. 33, no. 6, pp. 5240-5250, 2018.

[10] D. Rothmund, T. Guillod, D. Bortis, and J. W. Kolar, "99\% Efficient $10 \mathrm{kV}$ SiC-based $7 \mathrm{kV} / 400 \mathrm{~V}$ DC-Transformer for Future Data Centers," IEEE J. Emerg. Sel. Topics Power Electron. (early access), 2018.

[11] L. Heinemann, "An Actively Cooled High Power, High Frequency Transformer with High Insulation Capability," in Proc. of the IEEE Applied Power Electronics Conf. and Expo. (APEC), Mar. 2002.

[12] M. Leibl, G. Ortiz, and J. W. Kolar, "Design and Experimental Analysis of a Medium Frequency Transformer for Solid-State Transformer Applications," IEEE J. Emerg. Sel. Topics Power Electron., vol. 5, no. 1, pp. 110-123, 2017.

[13] F. Kieferndorf, U. Drofenik, F. Agostini, and F. Canales, "Modular PET, Two-Phase Air-Cooled Converter Cell Design and Performance Evaluation with 1.7kV IGBTs for MV Applications," in Proc. of the IEEE Applied Power Electronics Conf. and Expo. (APEC), Mar. 2016.

[14] T. Guillod, R. Färber, F. Krismer, C. M. Franck, and J. W. Kolar, "Computation and Analysis of Dielectric Losses in MV Power Electronic Converter Insulation," in Proc. of the IEEE Energy Conversion Congr. and Expo. (ECCE USA), Sep. 2016. 
[15] T. Guillod, J. E. Huber, G. Ortiz, A. De et al., "Characterization of the Voltage and Electric Field Stresses in Multi-Cell Solid-State Transformers," in Proc. of the IEEE Energy Conversion Congr. and Expo. (ECCE USA), Sep. 2014.

[16] S. B. Y. Du, G. Wang, and S. Bhattacharya, "Design Considerations of High Voltage and High Frequency Transformer for Solid State Transformer Application," in Proc. of the IEEE Industrial Electronics Society Conf. (IECON), Nov. 2010.

[17] T. B. Gradinger, U. Drofenik, and S. Alvarez, "Novel Insulation Concept for an MV Dry-Cast Medium-Frequency Transformer," in Proc. of the European Conf. Power Electronics and Applications (EPE), Sep. 2017.

[18] J. Jagers and S. Tenbohlen, "Evaluation of Transformer Reliability Data Based on National and Utility Statistics," in Proc. of the Int. Symp. on High Voltage Engineering, Aug. 2009.

[19] K. Niayesh and E. Gockenbach, "On the Aging Mechanism of Solid Insulating Materials Exposed to Repetitive High Voltage Pulses," IEEE Trans. Dielectr. Electr. Insul., vol. 21, no. 1, pp. 304-310, 2014.

[20] D. Fabiani, G. C. Montanari, and A. Contin, "Aging Acceleration of Insulating Materials for Electrical Machine Windings Supplied by PWM in the Presence and in the Absence of Partial Discharges," in Proc. of the IEEE Conf. on Solid Dielectrics (ICSD), Jun. 2001.

[21] D. Konig, N. Hardt, and V. Scherb, "Comparitive Insulation Tests with DC and $\mathrm{AC}$ at $50 \mathrm{~Hz}$ and $50 \mathrm{kHz}$," in Proc. of the IEEE Conf. on Electrical Insulation and Dielectric Phenomena (CEIDP), Oct. 1998.

[22] P. Wang, A. Cavallini, and G. Montanari, "The Influence of Repetitive Square Wave Voltage Parameters on Enameled Wire Endurance," IEEE Trans. Dielectr. Electr. Insul., vol. 21, no. 3, pp. 1276-1284, 2014

[23] T. Guillod, F. Krismer, and J. W. Kolar, "Electrical Shielding of MV/MF Transformers Subjected to High dv/dt PWM Voltages," in Proc. of the IEEE Applied Power Electronics Conf. and Expo. (APEC), Mar. 2017.

[24] S. Zhao, Q. Li, F. C. Lee, and B. Li, "High-Frequency Transformer Design for Modular Power Conversion From Medium-Voltage AC to 400 VDC," IEEE Trans. Power Electron., vol. 33, no. 9, pp. 7545-7557, 2018.

[25] F. Zhang, F. Z. Peng, and Z. Qian, "Study of the Multilevel Converters in DC-DC Applications," in Proc. of the IEEE Power Electronics Specialists Conf. (PESC), Jun. 2004.

[26] L. F. Costa, G. Buticchi, and M. Liserre, "Quad-Active-Bridge DC-DC Converter as Cross-Link for Medium-Voltage Modular Inverters," IEEE Trans. Ind. Appl., vol. 53, no. 2, pp. 1243-1253, 2017.

[27] H. Krishnaswami and N. Mohan, "Three-Port Series-Resonant DC-DC Converter to Interface Renewable Energy Sources With Bidirectional Load and Energy Storage Ports," IEEE Trans. Power Electron., vol. 24, no. 10, pp. 2289-2297, 2009.

[28] M. Neubert, A. Gorodnichev, J. Gottschlich, and R. W. De Doncker "Performance Analysis of a Triple-Active Bridge Converter for Interconnection of Future DC-Grids," in Proc. of the IEEE Energy Conversion Congr. and Expo. (ECCE USA), Sep. 2016.

[29] M. Stojadinović and J. Biela, "Modelling and Design of a Medium Frequency Transformer for High Power DC-DC Converters," in Proc. of the IEEE Energy Conversion Congr. and Expo. (ECCE Asia), May 2018.

[30] P. Wang, G. C. Montanari, and A. Cavallini, "Partial Discharge Phenomenology and Induced Aging Behavior in Rotating Machines Controlled by Power Electronics," IEEE Trans. Ind. Electron., vol. 61 no. 12, pp. 7105-7112, 2014

[31] M. Birle and C. Leu, "Breakdown of Polymer Dielectrics at High Direct and Alternating Voltages Superimposed by High Frequency High Voltages," in Proc. of the IEEE Conf. on Solid Dielectrics (ICSD), Jun. 2013.

[32] W. S. Zaengl, "Dielectric Spectroscopy in Time and Frequency Domain for HV Power Equipment I. Theoretical Considerations," IEEE Elect. Insul. Mag., vol. 19, no. 5, pp. 5-19, 2003.

[33] IEEE, "IEEE Recommended Practice for Testing Insulation Resistance of Electric Machinery," IEEE Std 43-2013, Tech. Rep., Mar. 2014.

[34] R. Bartnikas, Engineering Dielectrics: Measurement Techniques. Electrical Properties of Solid Insulating Materials. ASTM, 1987.

[35] B. Sonerud, T. Bengtsson, J. Blennow, and S. M. Gubanski, "Dielectric Heating in Insulating Materials Subjected to Voltage Waveforms with High Harmonic Content," IEEE Trans. Dielectr. Electr. Insul., vol. 16, no. 4, pp. 926-933, 2009.

[36] R. Färber and C. Franck, "Modular Arbitrary Waveform Dielectric Spectrometer for Aging Diagnostics of Recessed Specimens," in Proc of the IEEE Electrical Insulation and Dielectric Phenomena (CEIDP), Oct. 2016.

[37] R. Färber and C. M. Franck, "Modular High-Precision Dielectric Spectrometer for Quantifying the Aging Dynamics in (Sub-)Picofarad Polymeric Specimens," IEEE Trans. Dielectr. Electr. Insul., vol. 25, no. 3 pp. 1056-1063, 2018.
[38] R. Eichhorn, Engineering Dielectrics: Electrical Properties of Solid Insulating Materials: Molecular Structure and Electrical Behavior. ASTM, 1983.

[39] W. Liang, L. Raymond, L. Gu, and J. Rivas, “27.12MHz GaN Resonant Power Converter with PCB Embedded Resonant Air Core Inductors and Capacitors," in Proc. of the IEEE Energy Conversion Congr. and Expo. (ECCE USA), Sep. 2015.

[40] J. Choi, D. Tsukiyama, Y. Tsuruda, and J. Rivas, “13.56 MHz 1.3 kW Resonant Converter with GaN FET for Wireless Power Transfer," in Proc. of the IEEE Wireless Power Transfer Conf. (WPTC), May 2015

[41] K. C. Kao, Dielectric Phenomena in Solids. Academic Press, 2004.

[42] C. J. F. Böttcher and P. Bordewijk, Theory of Electric Polarization, Dielectrics in Time-Dependent Fields, Vol. II. Elsevier, 1978.

[43] W. B. Westphal and A. Sils, Dielectric Constant and Loss Data. Air Force Materials Laboratory, Air Force Systems Command, 1972.

[44] W. Wasylkiwskyj, Signals and Transforms in Linear Systems Analysis. Springer, New York, 2013.

[45] A. K. Jonscher, "Dielectric Loss under Transient Excitation," Journal of Physics C: Solid State Physics, vol. 11, no. 14, pp. 601-606, 1978.

[46] J. D. Menczel and R. B. Prime, Thermal Analysis of Polymers: Fundamentals and Applications. John Wiley \& Sons, 2014.

[47] G. H. Hardy and E. M. Wright, An Introduction to the Theory of Numbers. Oxford University Press, 1979, Ch. 18.

[48] Novocontrol Technologies, "Alpha-A, Technical Specification," Mar. 2010.

[49] von Roll, "Damisol 3418 API,” Nov. 2013.

[50] F. Kremer and A. Schönhals, Broadband Dielectric Measurement Techniques (10-6 Hz to $1012 \mathrm{~Hz}$ ). Springer, 2012.

[51] D. Rothmund, T. Guillod, D. Bortis, and J. W. Kolar, "99.1\% Efficient $10 \mathrm{kV}$ SiC-based Medium Voltage ZVS Bidirectional Single-Phase PFC AC/DC Stage," IEEE J. Emerg. Sel. Topics Power Electron. (early access), 2018.

[52] M. Mogorovic and D. Dujic, "100kW, 10kHz Medium Frequency Transformer Design Optimization and Experimental Verification," IEEE Trans. Power Electron., 2018.

[53] L. Dalessandro, F. da Silveira Cavalcante, and J. W. Kolar, "SelfCapacitance of High-Voltage Transformers," IEEE Trans. Power Electron., vol. 22, no. 5, pp. 2081-2092, 2007.

[54] COMSOL, "COMSOL Multiphysics 5.1 - Reference Manual," Apr. 2015.

[55] K. Venkatachalam, C. R. Sullivan, T. Abdallah, and H. Tacca, "Accurate Prediction of Ferrite Core Loss with Nonsinusoidal Waveforms using only Steinmetz Parameters," in Proc. of the IEEE Workshop on Computers in Power Electronics, Jun. 2002.

[56] A. Wichmann, "Two Decades of Experience and Progress in Epoxy Mica Insulation Systems ror Large Rotating Machines," IEEE Trans. Power App. Syst., vol. 102, no. 1, pp. 74-82, 1983.

[57] B. Hofmann, "Distribution Transformers and EMC," Sep. 2011, Siemens.

[58] C. Johansson and M. Robertsson, "Broadband Dielectric Characterization of a Silicone Elastomer," Journal of Electronic Materials, vol. 36, no. 9, pp. 1206-1210, 2007.

[59] Dow Corning, “TC4605 HLV, Thermally Conductive Encapsulant," Sep. 2014.

[60] Agilent Technologies, “Agilent 4294A Precision Impedance Analyzer, Operation Manual,” Feb. 2003.

[61] IET Labs, “Primary Standard Capacitors, 1404 Series,” Oct. 2013.

[62] C. Neusel, H. Jelitto, D. Schmidt, R. Janssen et al., "ThicknessDependence of the Breakdown Strength: Analysis of the Dielectric and Mechanical Failure," Journal of the European Ceramic Society, vol. 35, no. 1, pp. 113-123, 2015.

[63] H. B. Dwight, Tables of Integrals and Other Mathematical Data. Macmillan Company, 1966, Eqn. 418.

[64] F. B. Hildebrand, Introduction to Numerical Analysis. McGraw-Hill, New York, 1974, Ch. 5.8 .

[65] K. Ohta and H. Ishida, "Comparison among Several Numerical Integration Methods for Kramers-Kronig Transformation," Applied Spectroscopy, vol. 42, no. 6, pp. 952-957, 1988.

[66] B. Hirschorn and M. E. Orazem, "On the Sensitivity of the KramersKronig Relations to Nonlinear Effects in Impedance Measurements," Journal of The Electrochemical Society, vol. 156, no. 10, pp. 345-351, 2009. 


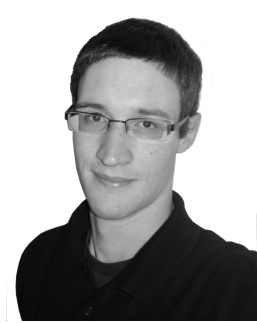

Thomas Guillod (S'14) received the M.Sc. degree in electrical engineering and information technology in 2013 from ETH Zurich, Switzerland with a focus on power electronics, numerical analysis, and field theory. In 2013, he joined the Power Electronic Systems Laboratory at ETH Zurich as a Ph.D. student and, in 2018, as a postdoctoral researcher. His current research interests include MV converters design, MF transformer optimization, electrical insulation in power converters, and numerical methods.

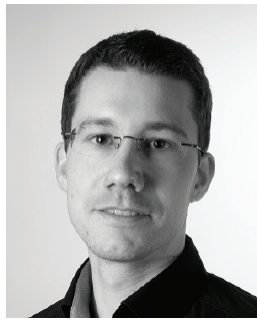

Raphael Faerber $\left(S^{\prime} 16\right)$ received a M.Sc. degree in physics from the Ecole Polytechnique Fédérale de Lausanne (EPFL), Switzerland. He is currently pursuing a $\mathrm{PhD}$ at the High Voltage Laboratory of ETH Zürich, Switzerland. His main research interests include charge transport and insulation degradation in polymeric insulators as well as the development of measurement systems for tracking the charge and degradation dynamics in high-voltage insulation materials/systems, with a particular focus on the medium-voltage insulation of power electronic converters.

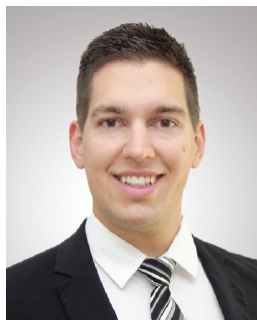

Daniel Rothmund (S'14) received the M.Sc. degree in electrical engineering and information technology from ETH Zurich, Switzerland, in 2013, with a focus on power electronics, high voltage technology, and electric power systems. In 2013, he joined the Power Electronic Systems Laboratory, ETH Zurich, as a Ph.D. student and received his Ph.D. degree in 2018. His current research interests include $10 \mathrm{kV}$ Silicon Carbide-based mediumvoltage AC to $400 \mathrm{~V}$ DC Solid-State Transformers and their optimization, calorimetric loss measurement methods, advanced medium-voltage insulation, and protection of $10 \mathrm{kV}$ Silicon Carbide devices. In 2019, he joined ABB Switzerland Ltd. as a scientist in the field of power electronics.



Florian Krismer (M'12) received the Dipl.-Ing. (M.Sc.) degree in electrical engineering with specialization in automation and control technology from the Vienna University of Technology, Vienna, Austria, in 2004, and the Ph.D. degree in electrical engineering from the Department of Information Technology and Electrical Engineering of ETH Zurich, Zurich, Switzerland, in 2010. He is currently a Research Associate at PES, where he has cosupervised Ph.D. students and has continued with his research in the field of power electronics. $\mathrm{He}$ is the author or coauthor of numerous conference and peer-review publications and has received two awards for his publications. His research interests include the analysis, design, and general optimization of power converter systems, e.g., the weight optimization of a bi-directional dcdc converter for an airborne wind turbine. Furthermore, he conducts research related to the filtering of conducted electromagnetic emissions and collaborated in the littlebox-challenge with respect to the hardware realization.

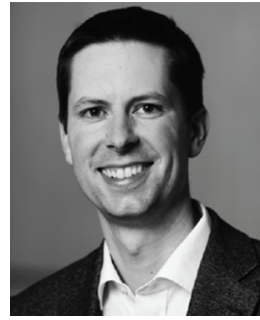

Christian M. Franck (SM'11) received the Diploma degree in physics from the University of Kiel, Kiel, Germany, in 1999, and the Ph.D. degree in physics from the University of Greifswald, Greifswald, Germany, in 2003. From 2003 to 2009, he was with the ABB Swiss Corporate Research Center, Baden Dättwil, Switzerland, as a Scientist and a Group Leader for gas circuit breakers and high-voltage systems. He is currently an Associate Professor of high-voltage technology with ETH Zürich, Zürich, Switzerland. His current research interests include gaseous and solid insulation systems, switching under HVDC conditions and the behaviour of water drops in electric fields.

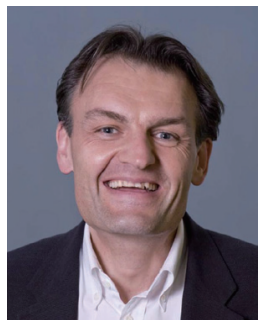

Johann W. Kolar (F'10) received his M.Sc. and Ph.D. degree (summa cum laude/promotio sub auspiciis praesidentis rei publicae) from the University of Technology Vienna, Austria, in 1997 and 1999 respectively. Since 1984, he has been working as an independent researcher and international consultant in close collaboration with the University of Technology Vienna, in the fields of power electronics, industrial electronics and high performance drive systems. He has proposed numerous novel PWM converter topologies, modulation and control concepts and has supervised 70+ Ph.D. students. He has published 880+ scientific papers in international journals and conference proceedings, 4 book chapters, and has filed 190+ patents. The focus of his current research is on ultra-compact and ultra-efficient $\mathrm{SiC}$ and $\mathrm{GaN}$ converter systems, solid-state transformers, advanced variable speed three-phase motor drives, integrated modular motor drives, ultra-high speed motors, bearingless motors/ actuators, and design automation in power electronics/mechatronics. Dr. Kolar has received 27 IEEE Transactions and Conference Prize Paper Awards, the 2014 IEEE Middlebrook Award, the 2016 IEEE William E. Newell Power Electronics Award, the 2016 IEEE PEMC Council Award and two ETH Zurich Golden Owl Awards for excellence in teaching. He initiated and/or is the founder of four ETH Spin-off companies. He is a member of the steering committees of several leading international conferences in the field and has served from 2001 through 2013 as an associate editor of the IEEE Transactions on Power Electronics. Since 2002 he also is an associate editor of the Journal of Power Electronics of the Korean Institute of Power Electronics and a member of the Editorial Advisory Board of the IEEJ Transactions on Electrical and Electronic Engineering. 